\title{
Ventral pallidal neurons code incentive motivation: amplification by mesolimbic sensitization and amphetamine
}

\author{
Amy J. Tindell, ${ }^{1}$ Kent C. Berridge,${ }^{1}$ Jun Zhang, ${ }^{1}$ Susana Peciña ${ }^{1}$ and J. Wayne Aldridge ${ }^{1,2}$ \\ ${ }^{1}$ Department of Psychology, University of Michigan, USA \\ ${ }^{2}$ Department of Neurology, University of Michigan Medical School, 1150 West Medical Center Drive, Medical Science Bldg I, Room \\ 3317, Ann Arbor, MI 48109-0607, USA
}

Keywords: addiction, amphetamine sensitization, incentive salience, neural coding, single-unit activity, Sprague-Dawley rat

\begin{abstract}
Neurons in ventral pallidum fire to reward and its predictive cues. We tested mesolimbic activation effects on neural reward coding. Rats learned that a Pavlovian conditioned stimulus $(C S+1$ tone) predicted a second conditioned stimulus ( $C S+2$ feeder click) followed by an unconditioned stimulus (UCS sucrose reward). Some rats were sensitized to amphetamine after training. Electrophysiological activity of ventral pallidal neurons to stimuli was later recorded under the influence of vehicle or acute amphetamine injection. Both sensitization and acute amphetamine increased ventral pallidum firing at CS+2 (population code and rate code). There were no changes at $\mathrm{CS}+1$ and minimal changes to UCS. With a new 'Profile Analysis', we show that mesolimbic activation by sensitization/amphetamine incrementally shifted neuronal firing profiles away from prediction signal coding (maximal at CS+1) and toward incentive coding (maximal at $\mathrm{CS}+2$ ), without changing hedonic impact coding (maximal at UCS). This pattern suggests mesolimbic activation specifically amplifies a motivational transform of CS+ predictive information into incentive salience coded by ventral pallidal neurons. Our results support incentive-sensitization predictions and suggest why cues temporally proximal to drug presentation may precipitate cue-triggered relapse in human addicts.
\end{abstract}

\section{Introduction}

The ventral pallidum (VP) is an output target of nucleus accumbens (Tang et al., 2005), tegmental dopamine neurons (Klitenick et al., 1992) and other limbic structures (Galaverna et al., 1993), and is involved in natural reward and drug addiction (Klitenick et al., 1992; Pierce \& Kalivas, 1997; Kalivas et al., 1999; Turner et al., 2002). Neurons in VP use population and firing rate codes to represent learned Pavlovian incentive cues that predict sucrose reward (Tindell et al., 2004). Here, we test the idea that VP neurons encode both predictive and motivational information about reward signals.

A conditioned stimulus $(\mathrm{CS}+)$ that predicts reward carries conditioned motivational value or incentive salience in addition to its predictive information (Toates, 1986). Amplification of this motivational (incentive) value by neural sensitization or psychostimulant administration is the basis for the incentive-sensitization theory of addiction (Robinson \& Berridge, 1993; Berridge \& Robinson, 1998), and may be a mechanism for CS-triggered relapse in human drug addicts (Dickinson \& Balleine, 2002; Robinson \& Berridge, 2003; McClure et al., 2003).

If the VP participates in the assignment of stimulus incentive value, its firing code should be amplified by mesolimbic manipulations, such as acute amphetamine or sensitization, which increase incentive salience of a reward CS+ (Wyvell \& Berridge, 2001). Here we compared predictive vs. incentive motivational impact of conditioned

Correspondence: Dr J. Wayne Aldridge, as above.

E-mail: jwaynea@umich.edu

Received 31 May 2005, revised 22 August 2005, accepted 25 August 2005 cues by presenting a series of two conditioned stimuli (CS+1 and $\mathrm{CS}+2$ ) followed by reward (unconditioned stimulus, UCS). The predictive impact and motivational impact of these stimuli are different. The first stimulus has more predictive information (Schultz et al., 1997). In contrast, the second (UCS-proximal) stimulus has more incentive value as motivation rises toward impending reward (Balleine et al., 1995; Corbit \& Balleine, 2003). We recorded VP neural activity elicited by these stimuli after drug-induced changes in mesolimbic activity by either sensitization or acute amphetamine administration.

We implemented a novel computational technique, 'Profile Analysis', to compare the relative VP neural coding of the two conditioned stimuli and the reward. Pure coding of learned prediction implies maximal firing to the first cue $(\mathrm{CS}+1)$ that predicts all that follows (Schultz, 2002). By contrast, incentive coding implies maximal firing to the second cue $(\mathrm{CS}+2)$ that is temporally closest to reward and occurs during highest motivation (Corbit \& Balleine, 2003). Finally, coding of the magnitude of actual reward values implies maximal firing to the sucrose reward itself (UCS). Profile Analysis provides a powerful way to test these alternatives. We found that individual VP neurons integrate predictive information, incentive salience and reward value, with profiles tending toward prediction in normal untreated animals. Mesolimbic activation (either by prior sensitization or by amphetamine administration at test) shifts the profiles of VP neurons toward incentive coding at the expense of prediction coding. Furthermore, the shift toward incentive coding was immediate and did not require relearning, which supports the incentive sensitization hypothesis. We suggest that in a sensitized state, incentive coding by 
VP neurons might mediate increased cue-triggered 'wanting' and could lead to the compulsive relapse of addiction, especially for drug cues that occur close in time to their reward.

\section{Materials and methods}

\section{Sensitization of incentive coding by VP units (Experiment 1)}

Animals

Twenty-one male Sprague-Dawley rats (290-340 g) were housed on an 08.00-20.00 h reversed light-dark schedule. Water and food were supplied ad libitum, except during Pavlovian training or testing sessions. Chow (20-25 g per day) was given immediately after training/test sessions (so rats remained motivated to obtain sucrose). The University Committee on the Use and Care of Animals approved all experimental methods.

\section{Experimental chambers}

All sessions (training, sensitization and test/recording) were conducted in the same $28 \times 35 \mathrm{~cm}$ plastic test chamber, containing red house lights, a pellet spout and dish, and two speakers that played CS tones. A video camera recorded behavior. A computer program ('Mtask', written in this laboratory) managed all stimulus presentations and recorded stimulus presentation times, while the program Recorder (Plexon, Dallas, TX, USA) recorded neural activity. The clocks for neural and video recordings and the behavioral task control were synchronized to allow an offline analysis for evaluating the correlation between neural firing and stimulus presentations or movements. The latter were identified in a frame-by-frame analysis of videotapes (Aldridge \& Berridge, 1998; Tindell et al., 2004).

\section{Preparatory magazine training and Pavlovian conditioning}

(Days 1-17)

We chose a Pavlovian conditioned approach paradigm. This design does not magnify conditioned responses and thus minimizes potential motor confounds from sensitized behavior that might also alter VP firing. Although behavioral Pavlovian approach conditioned responses to a water or sucrose dish are increased if sensitization is induced prior to training, when it can influence learning acquisition (Harmer \& Phillips, 1998; Taylor \& Jentsch, 2001; Phillips et al., 2002; Olausson et al., 2003), to our knowledge dish approach conditioned responses have never been reported to be increased if sensitization is induced after Pavlovian training. Our aim was to sensitize in a manner known to increase CS+ incentive salience (Wyvell \& Berridge, 2001) and test behavior later with a procedure in which sensitization would not markedly change the expression of conditioned motor responses. The results below suggest we achieved that combination.

In the first training step, each rat received three 2-min magazine training sessions, in which a sucrose pellet (the UCS) was presented in the sucrose dish on a fixed-interval 1-min schedule. This ensured that each rat obtained the pellet from the dish within a few seconds after the feeder click, which also served as CS+2 (see below).

Next, all rats were taught discriminative Pavlovian reward associations for 14 consecutive days. Two different auditory tones served as $\mathrm{CS}+1$ and CS-. One tone was pulsed and low in frequency $(400 \mathrm{~Hz}$, $10 \mathrm{~s}$ total of approximately $0.75 \mathrm{~s}$ on/off pulsed), and the other was continuous and high in frequency $(3800 \mathrm{~Hz}, 10 \mathrm{~s}$ duration). For half the rats, CS+1 was the high continuous tone and CS- was the low pulsed tone, and for other rats this assignment was reversed. The 10-s $\mathrm{CS}+1$ tone predicted both the $\mathrm{CS}+2$ click, which began at the end of $\mathrm{CS}+1$ and was caused by activation of the sucrose delivery mechanism, and the availability of sucrose pellet reward (UCS) $1 \mathrm{~s}$ later (Tindell et al., 2004). Thus, two serial CS+ events, a tone (CS+1) and a feeder click $(\mathrm{CS}+2)$, predicted reward (UCS). $\mathrm{CS}+1 / \mathrm{CS}+2 / \mathrm{UCS}$ pairings and $\mathrm{CS}-$ tones (without UCS) were presented 10 times each per training session in pseudorandom order on a variable interval, 2-min schedule. Rats could not predict the identity or exact timing of the next tone.

\section{Mesolimbic activation}

Neural sensitization induced by repeated intermittent psychostimulants and acute amphetamine administration may have overlapping mesolimbic neural and psychological incentive effects relevant to reward (Kalivas \& Nakamura, 1999; Kelley et al., 2003; McClure et al., 2003; Robinson \& Berridge, 2003). For example, neural effects of sensitization include potentiated amphetamine-induced dopamine activation and long-term neurochemical as well as structural changes in mesolimbic neurons (Paulson et al., 1991; Robinson \& Kolb, 1999; Roitman et al., 2002). Acute amphetamine causes increased dopamine and related catecholamine release (Kuczenski et al., 1997). Amphetamine and sensitization also share psychological effects such as increasing the incentive salience attributed to a reward $\mathrm{CS}+$, as assessed by increased cue-triggered 'wanting' in pure incentive versions of a Pavlovian-Instrumental Transfer paradigm (Wyvell \& Berridge, 2001). To assess overlapping consequences for VP coding, we used sensitization (induced between learning and testing) and acute amphetamine (administered on the day of testing) to independently activate mesolimbic modulation of signals relevant to reward.

\section{Amphetamine sensitization (Days 18-32)}

Rats were divided randomly into two groups: amphetamine sensitization $(n=10)$ and vehicle control $(n=11)$. Sensitization was conducted in the same environment as training and testing so that any contextual associative gating of sensitization expression would not prevent us from detecting sensitization effects during subsequent tests. Rats in the amphetamine sensitization group were given daily injections of escalating doses of D-amphetamine sulphate $(1-8 \mathrm{mg} / \mathrm{kg}$ i.p. in sterile saline) for a series of 14 consecutive days (Paulson et al., 1991). Sensitization group rats received $1 \mathrm{mg} / \mathrm{kg}$ amphetamine on the first day, and doses increased until rats were injected with $8 \mathrm{mg} / \mathrm{kg}$ on the final days [dose order (mg/kg): 1, 2, 2, 3, 3, 4, 4, 5, 5, 6, 6, 6, 8, $8]$. After each injection, rats were placed in the conditioning chamber and left for $1 \mathrm{~h}$ undisturbed. The vehicle control rats never received amphetamine, and instead received daily i.p. injections of $0.1 \mathrm{~mL} / \mathrm{kg}$ saline for 14 days, followed each day by $1 \mathrm{~h}$ in the conditioning chamber. After the end of the 14 daily injections, all rats spent 1 month in their home cages undisturbed to allow incubation of sensitization.

\section{Electrode implantation surgery}

After sensitization incubation, on approximately day 60-63, rats were implanted with recording electrodes aimed at posterior VP, a region able to mediate 'liking' and 'wanting' for food reward (Tindell et al., 2004; Smith \& Berridge, 2005). Rats were anaesthetized with $100 \mathrm{mg} / \mathrm{kg}$ ketamine and $10 \mathrm{mg} / \mathrm{kg}$ xylazine. Each rat had a multiwire recording electrode bundle implanted unilaterally in VP (L: 2.5; A: -1.00 ; D: 7.3). An electrode bundle consisted of eight $50-\mu \mathrm{m}$ tungsten wires, clustered within a region of approximately $1 \mathrm{~mm}$ (Tindell et al., 2004). Seven wires served as separate recording channels, and the eighth wire served as reference channel for differential recording. During surgery, neuronal activity was monitored as the electrode bundle was lowered into the brain to help guide 
accurate placement in VP (Tindell et al., 2004). Electrodes and mounting adapter were fixed in place with dental acrylic.

\section{Electrophysiological recording: Pavlovian CS+, CS- and UCS testing}

On day 69, rats had the first of four consecutive days of 30-min Pavlovian CS and UCS testing. As during the last day of training, $\mathrm{CS}+1$ nose-crossing responses constituted $75 \%$ of all dish entries during tones, indicating that rats remembered the Pavlovian associations. Each session had 10 presentations of $\mathrm{CS}+1$ and $\mathrm{CS}+2$ followed by UCS, and 10 presentations of CS- followed by nothing, under conditions identical to Pavlovian training 6 weeks earlier. Rats received saline injections $(0.1 \mathrm{~mL} / \mathrm{kg}$, i.p.; this kept procedures identical to subsequent drug test) before the first two test sessions, which tested the primary effects of post-learning sensitization on VP coding of stimuli in the absence of any direct drug effects (vehicle tests; Day 69-70).

\section{Amphetamine testing and washout}

On the third test day (amphetamine test; Day 71), an injection of D-amphetamine sulphate $(2 \mathrm{mg} / \mathrm{kg}$ in $0.1 \mathrm{~mL} / \mathrm{kg})$ was administered to all rats $15 \mathrm{~min}$ before testing to examine effects of acute pharmacological activation on VP neuronal responses and to confirm psychomotor sensitization of stereotypy. Finally, rats were retested on the fourth test day (washout vehicle retest; day 72), in conditions identical to the first two vehicle days.

\section{Histology}

Rats were anesthetized with an overdose of pentobarbital and then perfused transcardially. Brains were sliced in $40-\mu \mathrm{m}$ coronal sections and stained with Cresyl violet. Slices were examined microscopically to verify electrode locations in the posterior VP, which were plotted in a computerized stereotaxic atlas.

\section{Spike discrimination}

Waveforms of single neurons were discriminated from other neurons and from noise using an interactive computer program (Offline Sorter; Plexon). Single neural units were verified by their refractory periods in an autocorrelation histogram (NeuroExplorer, Nex Technologies). As a final discrimination procedure, we performed a cross-correlation analysis (NeuroExplorer, Nex Technologies) on all units to ensure that any unit recorded simultaneously on two electrode sites was counted only once in the analysis. Any remaining units that could not be separated from other neurons or noise were discarded.

\section{Neural responses}

VP responses were generally phasic, occurred as early as $100-150 \mathrm{~ms}$, had peaks within the first $500 \mathrm{~ms}$, and had durations of $200 \mathrm{~ms}$ or more (Fig. 1B and C). Thus, neurons were identified as 'responsive' to $\mathrm{CS}+1, \mathrm{CS}+2$ click, CS- or UCS if the firing rate in the first $500 \mathrm{~ms}$ after stimulus onset was significantly different from the firing rate in the baseline period 10 -s period before the CS tone $(P<0.05$ in Bonferroni-corrected $t$-tests). The 500-ms window for CS+1 and CStones began at tone onset, and for the $\mathrm{CS}+2$ click began at activation of the feeder click mechanism. The $500 \mathrm{~ms}$ UCS period began at the moment that rats typically acquired the sucrose pellet $(1 \mathrm{~s}$ after the $\mathrm{CS}+2$ click of the feeder).

\section{Population coding}

Responsive neurons were counted to formulate tallies of units responsive to $\mathrm{CS}+1, \mathrm{CS}+2, \mathrm{CS}-$ or $\mathrm{UCS}$. We used chi-square tests for comparative testing across stimuli, across sensitization and control groups, and across vehicle test and amphetamine test conditions. Additional comparisons were made between proportions of excitatory and inhibitory responses, and between proportions of unique (responsive to one stimulus) and combination (responsive to more than one stimulus) responses. To eliminate any spurious contributions (e.g. a response due to increase in firing in only a few trials), we inspected every peri-event time histogram and raster plot visually.

\section{Neural rate coding}

Changes in firing rate elicited by $\mathrm{CS}+1, \mathrm{CS}+2$ and UCS were computed for each unit. The changes were normalized by dividing the absolute firing rate during the first $500 \mathrm{~ms}$ of each CS or UCS stimulus period by the firing rate during the baseline period immediately preceding the first stimulus in each trial. An additional analysis of normalized firing rates was performed over the entire $10 \mathrm{~s}$ duration of both tones. ANOVA and post-hoc Bonferroni tests were used for comparisons. The normalized firing rates computed in this analysis were used for the following Profile Analysis. All statistical tests and all graphs include all recorded VP neurons, whether responsive or non-responsive, excitatory or inhibitory, unless otherwise stated.

\section{Comparing predictive, incentive value and reward coding}

In our neural analysis, we compared the predictive vs. incentive values of conditioned stimuli for reward. The first conditioned stimulus in our series $(\mathrm{CS}+1)$ predicted all other conditioned and unconditioned stimuli that follow, and thus axiomatically $\mathrm{CS}+1$ had the most predictive value (Schultz et al., 1997). The second conditioned stimulus $(\mathrm{CS}+2)$, which was closer in time or temporally proximal to UCS, was considered to have the most incentive value because previous studies have shown that the incentive impact of Pavlovian cues gradually rises and focuses as $\mathrm{CS}+\mathrm{s}$ become more temporally proximal reward. For example, if a $\mathrm{CS}+$ predicts food reward within $2 \mathrm{~s}$, hungry rats proceed directly to the food dish, but if the same CS+ predicts food at a longer $6 \mathrm{~s}$ delay, the rats investigate the CS itself before going to the dish instead of directly approaching the food dish (Timberlake et al., 1982). Similarly, if a series of three CSs (10 s each) predict food reward for pigeons, the first CS+ elicits only general locomotion, but the third and final CS+ elicits consummatory key peck behaviors more appropriate to the food reward (Matthews \& Lerer, 1987). Finally, in a Pavlovian-Instrumental Transfer experiment, if a serial chain of two instrumental actions produces food reward, the ability of a reward CS + to elicit increased cue-triggered 'wanting' and spur instrumental performance was restricted to the second action in the series most proximal to reward (Corbit \& Balleine, 2003). Together such results support the idea that conditioned incentive salience and motivation rises when reward is expected, and peaks just before UCS delivery. Higher incentive salience may be an explanation for why a $\mathrm{CS}+2$ is less affected than a more distant $\mathrm{CS}+1$ by temporal discounting (i.e. the weakening of stimulus impact due to delays until reward; Vuchinich \& Simpson, 1998; Critchfield \& Kollins, 2001). We exploited the higher conditioned incentive value of a $\mathrm{CS}+2$ relative to $\mathrm{CS}+1$ in this experiment by using $\mathrm{CS}+1$ as a marker for greatest predictive value, $\mathrm{CS}+2$ as a marker for greatest incentive motivational value, and sucrose UCS as a marker for greatest reward impact in assessing VP coding functions.

\section{Analysis of neural response profiles}

We used a novel analysis of neural response profiles to assess integrative coding properties of all 524 VP neurons. By integrative 

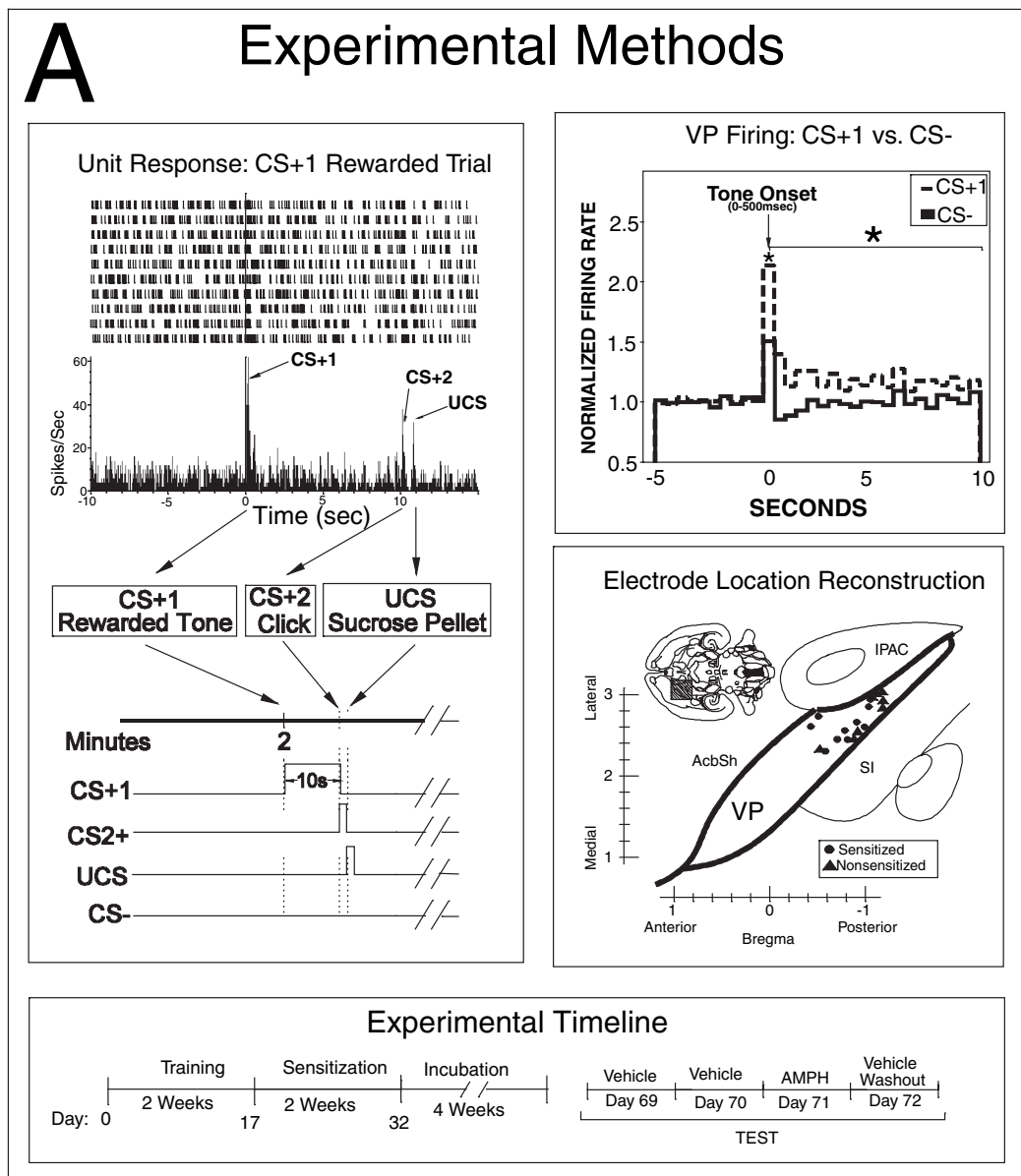

B Sensitization Effect
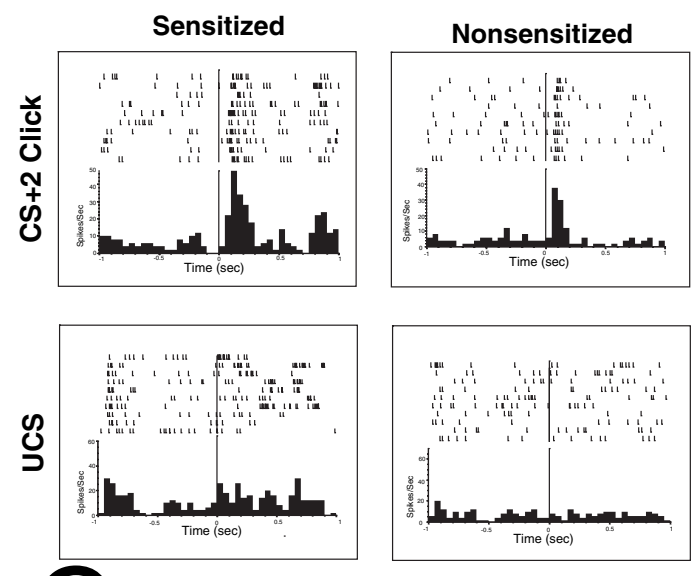

0

Amphetamine Effect
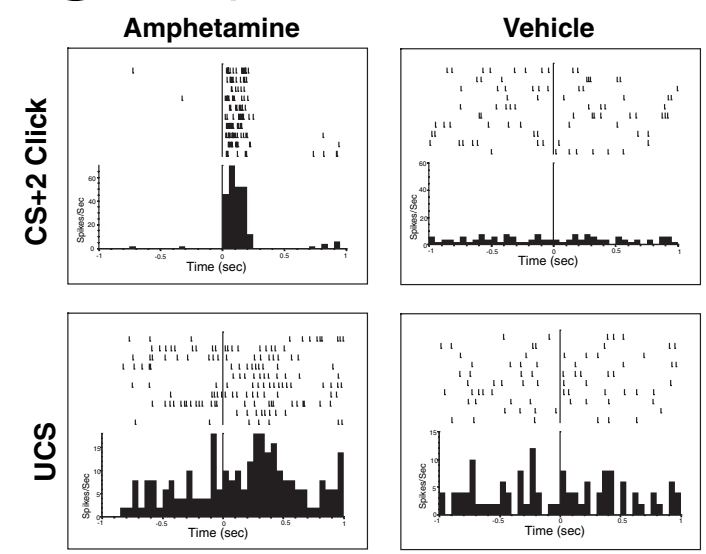

FIG. 1. Schematic of experimental methods (left) and examples of sensitization and amphetamine effects on incentive conditioned stimulus (CS+) and sucrose unconditioned stimulus (UCS) coding (right). (A) A ventral pallidum (VP) neuron responds to CS +1 tone, CS +2 click and sucrose UCS, shown with a raster and histogram (top left). Each mark in a raster line indicates a spike, and each line indicates a trial, with consecutive trials in one test going from top to bottom. Histograms show the firing rate averaged across all trials. Timing and order of CS $+1, \mathrm{CS}+2$ and UCS presentation are shown by line blocks below (bottom left). VP firing during CS +1 and CS - tones are compared in an overlapping histogram reflecting all recorded neurons (top right; $P<0.05$ ). All electrode locations were in posterior VP as intended (horizontal atlas map; bottom right). Experimental timeline shows chronology of training, sensitization, incubation and test phases (bottom). (B) Sensitization increases VP firing to CS +2 and subsequent UCS (shown for four VP neurons in sensitized vs. non-sensitized rats on first test day). (C) Acute amphetamine administration (test 3) also increases VP firing to CS+2 and UCS, but vehicle (tests 1,2 and 4) does not. Acute amphetamine administration also increases VP firing to CS+2 and UCS.

coding, we mean that a VP neuron may respond to more than one stimulus (CS $+1, \mathrm{CS}+2$, UCS, etc.) rather than being dedicated to only one, yet still respond differently to each stimulus and have uniquely highest firing to a single particular stimulus. Profile Analysis captures these complex firing relations by creating a quantitative index (a vector) representing the relative order of magnitudes of a neuron's firing rates to the three reward stimuli, $\mathrm{CS}+1, \mathrm{CS}+2$ and UCS. The computation is such that index values exist in a continuum that: (i) exhausts all possible firing patterns (i.e. relative orders in firing rates) to these three stimuli; and (ii) guarantees that nearby values represent similar firing patterns. The rationale is derived from a method proposed earlier (called 'Locus Analysis') to characterize sensorimotor transformation roles of neurons in primary motor cortex (Zhang et al., 1997).

We denote each neuron's firing rate (normalized to baseline) to $\mathrm{CS}+1, \mathrm{CS}+2$ and $\mathrm{UCS}$ as $x, y, z$, respectively. The relative rankordering of these three numbers according to their magnitude represents the 'profile' of a neuron's responses to the stimuli. This response profile can be represented mathematically as a vector in a two-dimensional space. For each neuron we constructed a twodimensional vector $(\alpha, \beta)$ where

$$
\begin{gathered}
\alpha=(2 y-x-z) / 2 \\
\beta=\sqrt{ } 3(x-z) / 2
\end{gathered}
$$

The $(\alpha, \beta)$ components capture the two orthogonal contrasts formed among the three dependent variables $x, y, z$, such that any other contrast is a rotation in the two-dimensional space. This vector's magnitude

$$
r=\sqrt{ }\left(\alpha^{2}+\beta^{2}\right)=\sqrt{ }\left[(x-y)^{2}+(y-z)^{2}+(z-x)^{2}\right] / 2
$$

represents the extent to which the neuron's firing rates, $x, y, z$ are differentially modulated by the three types of stimuli $(\mathrm{CS}+1, \mathrm{CS}+2$ 
and UCS); it actually represents the variance of responses across the stimuli. The vector's direction

$$
\theta=\tan ^{-1}(\beta / \alpha)
$$

reflects the rank-ordering of the magnitudes of these firing rates in the unit's response profile. For instance, when $\theta=0^{\circ}$, corresponding to $\alpha>0, \beta=0$, this indicates a profile with $y>x=z$, that is, neuronal firing to $\mathrm{CS}+2$ is faster than firing to $\mathrm{CS}+1$ and $\mathrm{UCS}$, while the latter two firing rates are equal. Analogously, when $\theta=180^{\circ}$, corresponding to $\alpha<0, \beta=0$, this profile indicates $x=z>y$, that is, neuronal firing to $\mathrm{CS}+2$ is slower than firing to either CS+1 or UCS (the latter two rates are equal). Using similar reasoning, it can be seen that $\theta=60^{\circ}, 120^{\circ}, 240^{\circ}\left(-120^{\circ}\right)$ and $300^{\circ}\left(-60^{\circ}\right)$ correspond to the firing profiles of, respectively, $x=y>z, x>y=z, z>x=y$, and $y=z>x$. These are the boundary lines for the six rank-orders associated with three arbitrary numbers $x, y, z$. Note that directions identified in this way capture the neuronal firing profile in a continuum of angles; this procedure is both exhaustive (i.e. all neurons can be characterized) and faithful (i.e. the distance between the angles monotonically reflects the difference in two profiles).

Each unit's profile vector was calculated based on responses to the three stimuli (CS+1, CS +2 , UCS) as described above. For comparing populations, we computed distributions of neurons in profile space and Population Profile Vectors. Distributions were determined by counting the number of unit profile vectors in each of 12 bins $30^{\circ}$ wide (the $0^{\circ}$ bin consists of all those units whose directional angle is between $-15^{\circ}$ and $15^{\circ}$, the $90^{\circ}$-bin consists of all those units whose angles are between $75^{\circ}$ and $105^{\circ}$, etc.). Population Profile Vectors were computed as the vector sum of individual unit profile vectors, i.e. $(\alpha, \beta)$ values. The Population Profile Vector represents the overall population responses to the three stimuli $(\mathrm{CS}+1, \mathrm{CS}+2$, UCS). Like the constituents, the angular directions of the Population Profile Vector reflects the relative rank-order of the magnitude of the firing responses to these stimuli, whereas vector lengths represent the variance of the responses across the three stimuli. For statistical comparison of Population Profile Vectors, we employed multivariate ANOVA in SPSS (Figs 5-7).

Of particular interest to this study are the profile regions where each of the stimuli dominates (Fig. 5). Specific reward learning models lead to distinct hypotheses about distributions of response profiles and Population Profile Vector properties. Prediction signal coding anticipates profiles with $\mathrm{CS}+1>\mathrm{CS}+2>\mathrm{UCS}$ for the relative ordering of response magnitudes. Thus, the region spanning $60^{\circ}-180^{\circ}$ is where $\mathrm{CS}+1$ dominates the response profile and represents neurons that are responsive to the cue that is most unpredictable. This is designated as 'predictive signal coding'. On the other hand, incentive salience theory anticipates $\mathrm{CS}+2>$ $\mathrm{CS}+1>\mathrm{UCS}$ for incentive-coding neurons that motivationally transform the learned CS+ most proximal to the reward, i.e. CS+2. The region spanning $-60^{\circ}$ to $60^{\circ}$ represents dominant neural firing to $\mathrm{CS}+2$, and we denote it as the motivational or 'incentive-coding' area. Finally, UCS value-coding neurons should have profiles with $\mathrm{UCS}>\mathrm{CS}+2>\mathrm{CS}+1$. The region spanning $-180^{\circ}$ to $-60^{\circ}$ represents dominance by the reward itself (UCS), and it is designated the 'UCS value-coding' profile region (i.e. UCS hedonic impact or associative stamping-in/teaching signal).

\section{Control for motor-related VP activity during Pavlovian tests}

To determine whether neural activity of responsive VP units during CS tones was simply explained by concurrent movements, we examined movement-related activation of units responsive to $\mathrm{CS}+1$ or CS- during head movements, mouth movements, and forelimb and locomotion movements that occur during spontaneous approachtype responses and grooming. This analysis was performed for sensitized and non-sensitized rats in both vehicle and amphetamine challenge conditions. We especially examined spontaneous head shift movements and left and right forelimb steps because most rats responded to $\mathrm{CS}$ tones with these movements ( $\mathrm{CS}+1, \mathrm{CS}-)$. In addition, we examined movements during the baseline periods before tones, i.e. before any signal that trials had even begun. We performed an offline frame-by-frame analysis of videotape segments in which the animal was adequately in the field of view for assessment. We evaluated movements in response to CS tones [63\% of units responded to CS+ (140/223); $65 \%$ of units responded to CS- (102/157)]. Additionally for 50\% (82/165) of units responsive to UCS, we examined mouth and tongue movement-related activity during grooming to determine whether jaw or tongue movements activated these cells. Peri-movement histograms were computed in the same manner as those used to assess conditioned stimuli to identify possible significant movement-related activity of cueresponsive units.

\section{Behavioral conditioned approach}

Conditioned approach responses to the sucrose dish, elicited by $\mathrm{CS}+\mathrm{s}$ or CS-, were measured by a touch detector, signaling that the rat's nose had entered the sucrose dish. The number of contacts elicited during a $\mathrm{CS}+1$ or $\mathrm{CS}-10$-s tone was compared with the number of contacts occurring during a preceding baseline 10 -s period before that tone. Baseline values were subtracted from $\mathrm{CS}+1$ and $\mathrm{CS}-$ values to produce difference scores (to control for possible changes in baseline activity across sessions). ANOVA and Bonferroni-corrected post-hoc tests (SPSS; Chicago, IL, USA) were used for analyses.

\section{Behavioral evaluation of amphetamine-stereotypy}

Behavioral sensitization of psychomotor stereotypy was assessed during amphetamine and vehicle test sessions. Locomotion and stereotypic behavior session was scored for every test in an off-line video analysis using the following scale: 1, inactive; 2, stationary activity; 3, pellet activity; 4, mobile regular activity; 5, hyperactivity; 6, occasional stereotypic head and sniffing movements; 7, continual sniffing in a wide area; 8 , intense focused stereotypic movements; 9, intense focused stereotypic movements at sucrose dish (MacLennan \& Maier, 1983). CS-related stereotypy ratings were made for each $\mathrm{CS}+1$ and $\mathrm{CS}$ - tone, and baseline ratings were made once a minute. Averages were computed for each rat and test session.

\section{Experiment 2: behavioral sensitization of taste 'liking' measured by affective taste reactivity}

In order to rule out whether any VP changes in UCS coding reflected increased hedonic impact of the sucrose UCS, we used the affective taste reactivity measure of 'liking' reactions elicited by oral infusions of sucrose in a separate experiment to assess sensitization and acute amphetamine effects. Sweet vs. bitter tastes elicit several behavioral affective reactions from rats that are homologous to affective facial expressions of human infants, great apes and monkeys (e.g. rhythmic tongue protrusions to sucrose; aversive gapes to quinine; Berridge, 2000; Steiner et al., 2001). Taste reactivity measures can help assess whether sensitization or amphetamine administration increase the 'liking' reaction or hedonic impact elicited by a sucrose UCS. 
Twenty-eight additional rats were randomly assigned to three groups: eight rats received the sensitization regimen exactly as described above, another eight rats received a sensitization regimen of $3 \mathrm{mg} / \mathrm{kg}$ amphetamine repeated daily for 6 days (used in a previous study that demonstrated sensitization of $\mathrm{CS}+$ incentive salience; Wyvell \& Berridge, 2001), and 12 rats received only vehicle control injections. A sensitization incubation period of 1 month was allowed after the last injection before surgery and testing. All rats were anesthetized and implanted with oral cannulae (heat-flared PE-100 tubing) that entered the mouth lateral to the first maxillary molar to permit oral infusions for taste reactivity testing. In addition, the second sensitization group also received implantation of bilateral microinjection cannulae in nucleus accumbens shell in the same surgery (+ $3.1 \mathrm{~mm}$ bregma; $+1.0 \mathrm{~mm}$ lateral; $5.7 \mathrm{~mm}$ ventral; incisor bar set at $+5.0 \mathrm{~mm}$ ). Microinjection of amphetamine was used in this group to further disentangle any potentially confounding sensorimotor effects of systemic amphetamine from sucrose hedonic impact. For example, if amphetamine acts in neostriatum or brainstem to suppress motor expression of 'liking' reactions, then systemic amphetamine is not a good way to test if increased mesolimbic dopamine enhances 'liking'. Instead, use of amphetamine microinjection here allowed us to selectively test if mesolimbic dopamine increases 'liking' reactions (and VP coding of 'liking') in control or sensitized rats, while avoiding potential confounds introduced by systemic injections given at the time of test.

After 1 week recovery, taste reactivity tests were performed in which a $1 \mathrm{~mL}$ volume of either $0.1 \mathrm{M}$ sucrose or $3 \times 10^{-5} \mathrm{M}$ quinine $\mathrm{HCl}$ solutions were infused into a rat's mouth through the oral cannula by a syringe pump over a period of $1 \mathrm{~min}$. Prior to each taste reactivity test, rats were injected either with amphetamine or vehicle (i.p.; first group), or were microinjected in nucleus accumbens with either amphetamine or vehicle ( 0 or $20 \mu \mathrm{g}$ in $0.5 \mu \mathrm{L}$; second group). Thus, over four test days, rats received only one taste infusion (sucrose or quinine) per day and only one injection (amphetamine or vehicle) per day, and the order of taste/drug testing was counterbalanced across rats.

Affective reaction patterns were scored in slow motion video analysis $(1 / 30 \mathrm{~s}$ frame-by-frame to $1 / 10$ actual speed; using bout scoring criteria described in Berridge, 2000). Positive hedonic reactions included rhythmic midline tongue protrusions, lateral tongue protrusions and paw licking. Negative aversive reactions included gapes, headshakes, forelimb flails, face washing, chin rubs and paw treading.

\section{Results \\ Sensitization and amphetamine increase VP firing to $C S+2$ (Experiment 1)}

\section{General}

VP neurons responded to the Pavlovian $\mathrm{CS}+1, \mathrm{CS}+2$ and UCS stimuli that rats had learned 6 weeks earlier. Rats also responded behaviorally to $\mathrm{CS}+$ stimuli, in that they showed conditioned approach to the sucrose dish, reaching it about $4 \mathrm{~s}$ after the onset of the 10 -s CS +1 . That meant they were already actively investigating the dispenser spout when the $0.5-\mathrm{s} \mathrm{CS}+2$ occurred. Once at the dish, rats typically made increasingly more frequent nose pokes as the CS+2 and UCS approached. This ramping up of dish-nose poke probability throughout the tone meant that at the moment of $\mathrm{CS}+2$ (click), rats were most likely to have their mouths positioned in the sucrose dish, waiting open and ready to catch the sucrose pellet UCS $1 \mathrm{~s}$ later (Fig. 1A). This ramping pattern of nose-poke behavior at the dish suggests increasing motivation from $\mathrm{CS}+1$ to $\mathrm{CS}+2$.

\section{Histology}

Electrodes of 18 rats (10 sensitized, eight control) were confirmed to be located as intended in posterior ventral pallidum $(\mathrm{AP}=-0.4$ to -1.08 Bregma; $\mathrm{ML}=2.2$ to $3.2 ; \mathrm{DV}=-7.2$ to -8 ; Fig. $1 \mathrm{~A}$ ).

\section{Responsive VP neurons: population sizes}

During four test sessions we recorded 524 VP neural units (246 sensitized, 278 non-sensitized). VP neurons were identified as responsive to $\mathrm{CS}+1$ tone, $\mathrm{CS}+2$ click, $\mathrm{CS}-$ tone, or UCS reward if the firing rate during their first $500 \mathrm{~ms}$ was significantly above or below baseline firing during the 10 -s period before its CS tone. Of the $524 \mathrm{VP}$ units tested in all rats across the four tests, 278 units (53\%) responded to $\mathrm{CS}+1 ; 298$ units $(57 \%)$ to $\mathrm{CS}+2$; and 194 units $(37 \%)$ to CS- (Table 1). Thus, similar majorities of VP neurons responded to the two CS+ stimuli, whereas CS-, which signaled nothing, evoked fewer neurons, and the sucrose UCS evoked a population of 78 units $(34 \%)$ that was similar in size to the CS- $(\mathrm{CS}+1[53 \%$ of units $]=\mathrm{CS}+2[57 \%])>(\mathrm{UCS}[34 \%]=\mathrm{CS}-[37 \%])$. There was no statistically significant difference between the $\mathrm{CS}+1$ and $\mathrm{CS}+2$ populations, whereas there were significantly fewer $\mathrm{CS}-$ and UCS responses $(\mathrm{CS}+1=\mathrm{CS}+2[P=0.701]$; $\mathrm{CS}+1$ or $\mathrm{CS}+2>\mathrm{CS}-[$ both $P<0.0001] ; \mathrm{CS}+1$ tone or $\mathrm{CS}+2>\mathrm{UCS}$ reward [both $P<0.0001$; $\mathrm{UCS}=\mathrm{CS}-[P=0.994])$.

TABLE 1. Responsive neurons (total $n=524$ ) from sensitized and non-sensitized rats during vehicle and amphetamine tests

\begin{tabular}{|c|c|c|c|c|c|c|c|c|}
\hline & \multicolumn{2}{|c|}{$\begin{array}{l}\text { Test } 1(18 \%) \\
\text { Sensitized }(n=44) \\
\text { Non-sensitized }(n=49)\end{array}$} & \multicolumn{2}{|c|}{$\begin{array}{l}\text { Test } 2(27 \%) \\
\text { Sensitized }(n=69) \\
\text { Non-sensitized }(n=75)\end{array}$} & \multicolumn{2}{|c|}{$\begin{array}{l}\text { Test } 3(25 \%) \\
\text { Sensitized }(n=58) \\
\text { Non-sensitized }(n=73)\end{array}$} & \multicolumn{2}{|c|}{$\begin{array}{l}\text { Test } 4(30 \%) \\
\text { Sensitized }(n=75) \\
\text { Non-sensitized }(n=81)\end{array}$} \\
\hline & Excitatory & Inhibitory & Excitatory & Inhibitory & Excitatory & Inhibitory & Excitatory & Inhibitory \\
\hline \multicolumn{9}{|c|}{ Sensitized $(n=246,47 \%)$} \\
\hline $\mathrm{CS}+1$ & $26(59 \%)$ & $0(0 \%)$ & $27(39 \%)$ & $4(6 \%)$ & $22(38 \%)$ & $1(2 \%)$ & $42(56 \%)$ & $1(1 \%)$ \\
\hline $\mathrm{CS}-$ & $14(32 \%)$ & $1(2 \%)$ & $21(30 \%)$ & $4(6 \%)$ & $9(16 \%)$ & $8(14 \%)$ & $20(27 \%)$ & $7(9 \%)$ \\
\hline $\mathrm{CS}+2$ & $26(59 \%)$ & $1(2 \%)$ & $46(67 \%)$ & $4(6 \%)$ & $37(64 \%)$ & $1(2 \%)$ & $44(59 \%)$ & $1(1 \%)$ \\
\hline UCS & $15(34 \%)$ & $1(2 \%)$ & $27(39 \%)$ & $7(6 \%)$ & $15(26 \%)$ & $4(7 \%)$ & $18(24 \%)$ & $2(3 \%)$ \\
\hline \multicolumn{9}{|c|}{ Non-sensitized $(n=278,53 \%)$} \\
\hline $\mathrm{CS}+1$ & $22(45 \%)$ & $3(6 \%)$ & $38(51 \%)$ & $6(8 \%)$ & $30(41 \%)$ & $1(1 \%)$ & $43(53 \%)$ & $10(12 \%)$ \\
\hline CS- & $14(29 \%)$ & $4(8 \%)$ & $18(24 \%)$ & $5(7 \%)$ & $22(30 \%)$ & $5(7 \%)$ & $25(31 \%)$ & $14(17 \%)$ \\
\hline $\mathrm{CS}+2$ & $19(39 \%)$ & $2(4 \%)$ & $38(51 \%)$ & $7(9 \%)$ & $38(52 \%)$ & $8(11 \%)$ & $34(42 \%)$ & $5(6 \%)$ \\
\hline UCS & $8(16 \%)$ & $11(22 \%)$ & $13(17 \%)$ & $7(9 \%)$ & $13(18 \%)$ & $8(11 \%)$ & $19(23 \%)$ & $10(12 \%)$ \\
\hline
\end{tabular}

Absolute numbers of recorded neurons and percentages (in parentheses) are shown for each test day. 
Sensitization and acute amphetamine effects on population coding. We found that sensitized rats had more units responsive to $\mathrm{CS}+2$ than non-sensitized rats overall (chi square: $P<0.05$; Fig. $2 \mathrm{~A}$ ). The increase was specific to $\mathrm{CS}+2$ click, as sensitization had no effect on the proportions of responsive neurons to stimuli overall (chi square: $P>0.05$; Fig. 2A). The sizes of neuronal populations that responded to $\mathrm{CS}+1, \mathrm{CS}-$ or UCS were not altered by sensitization. Amphetamine challenge had no overall effect on the sizes of activated VP neural populations to any stimulus (chi square: $P>0.05$ ).

Multiple stimuli vs. unique stimulus. Of the 427 responsive units, the majority $(73 \%, n=311 / 427)$ responded to more than one stimulus. For example, $71 \%(n=198 / 276)$ of $\mathrm{CS}+1$ units also responded to $\mathrm{CS}+2$. The most common activation pattern was a brief peak $(<2 \mathrm{~s})$ of increased firing at the onset of CS+1 and another brief peak at the $\mathrm{CS}+2$ click $(n=125$; Fig. $1 \mathrm{~A})$. Further, $32 \%$ of $\mathrm{CS}+1$ units ( $n=87 / 276$ ) also responded to UCS, in addition to responding to the intervening $\mathrm{CS}+2$, thus responding to all three reward stimuli in series. The majority of UCS units also responded to CS+2 $(68 \%$, $n=121 / 178$ ).

For the CS- tone that signaled no reward, the most common activation pattern was a brief increase at tone onset $(<2 \mathrm{~s} ; n=68)$. Some generalization may have occurred in VP coding of tone conditioned stimuli (though $\mathrm{CS}+$ and $\mathrm{CS}-$ tones were well discriminated behaviorally and in VP firing rates), in that most units that responded to $\mathrm{CS}-$ also responded to $\mathrm{CS}+1$ or $\mathrm{CS}+2 \quad(85 \%$, $n=164 / 192$ ). Additionally, 94 units showed a change in activity at CS- tone offset, when no stimulus was actually presented, but about when the UCS would have occurred on a rewarded CS +1 trial.

One hundred and sixteen neurons $(27 \%)$ responded to only one stimulus during the four tests. The $\mathrm{CS}+2$ had twice as many unique responsive neurons ( $n=47$ or $41 \%$ unique responses) as the CS +1 $(P<0.001 ; n=23$ or $20 \%)$, the CS- $(P<0.001 ; n=23$ or $20 \%)$ or the UCS $(P<0.001 ; n=23$ or $20 \%)$. Neither sensitization nor test session affected these relative distributions of neuronal firing to particular stimuli $(P>0.05$ for both).
A Population Code: Sensitization Effect

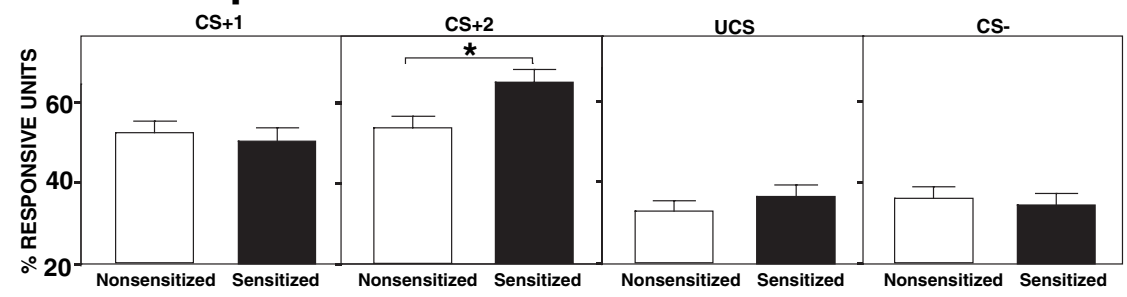

B Rate Code: Sensitization Effect

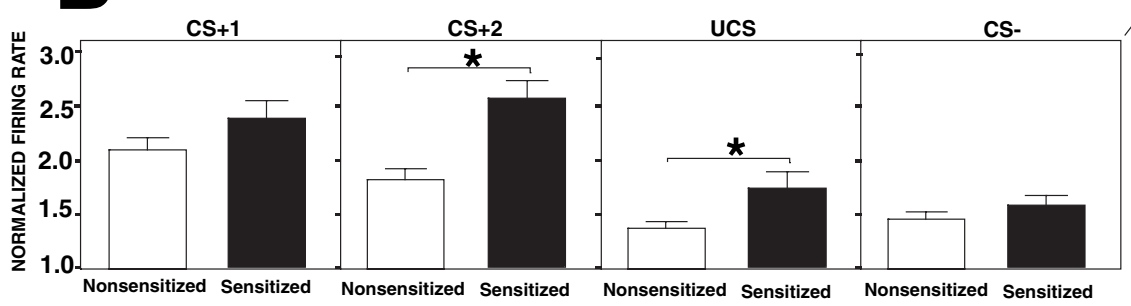

Date Code: Acute Amphetamine Effect

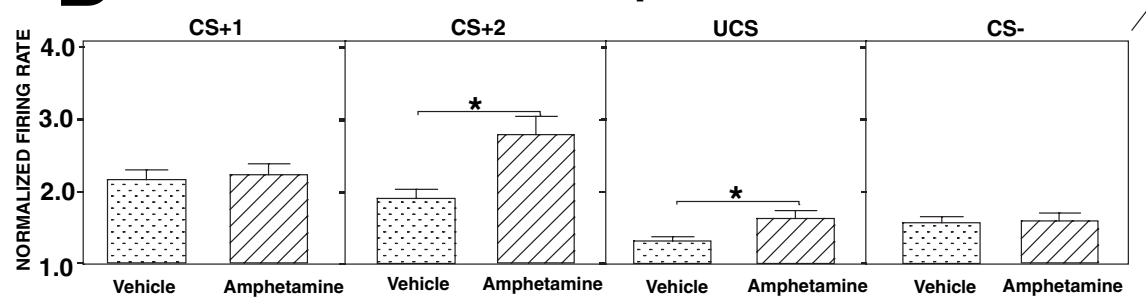

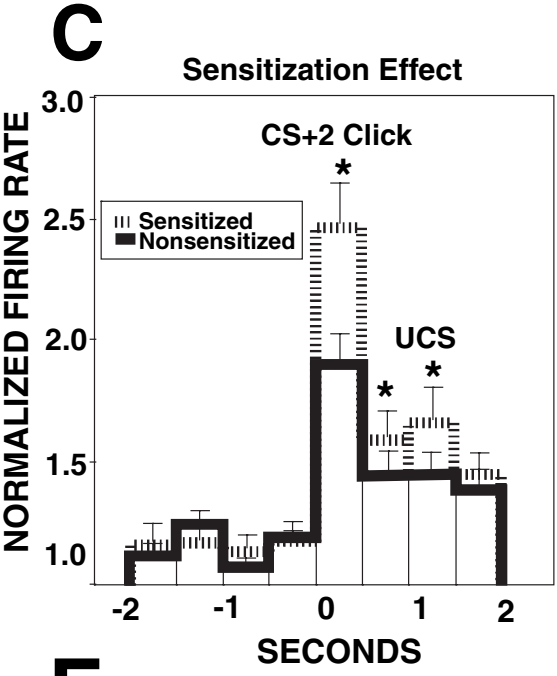
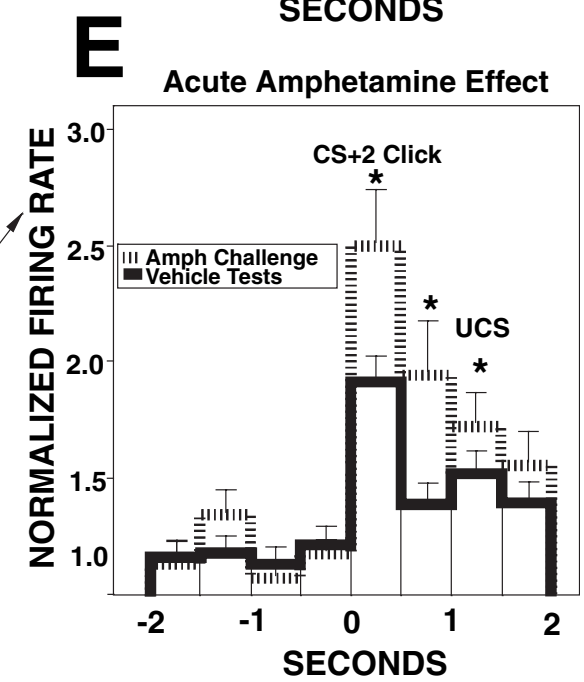

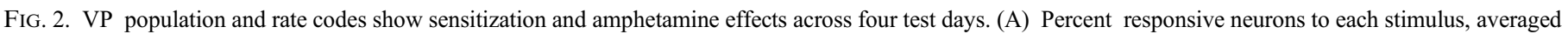

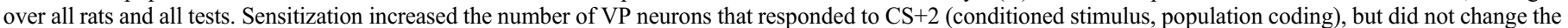

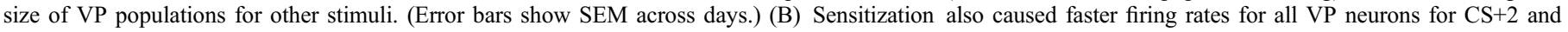

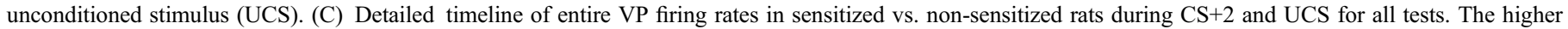

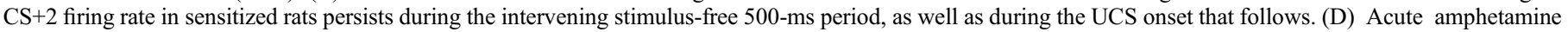

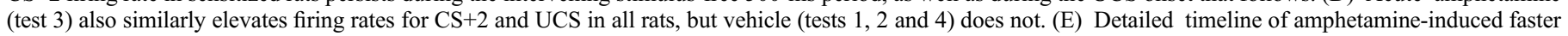
firing rates during $\mathrm{CS}+2$, intervening $500 \mathrm{~ms}$ interval, and subsequent UCS for entire VP population. ${ }^{*} P<0.05$, comparing groups. 
Excitatory vs. inhibitory population responses. We observed both excitatory and inhibitory responses to conditioned stimuli and sucrose UCS, and sometimes both types were observed from the same unit to different stimuli ( $n=69,20 \%$ of units responsive to multiple stimuli). The majority of responses on all tests were excitatory (770 excitatory responses or $81 \%$ vs. 143 inhibitory responses or $19 \%$; observed in 427 units). The CS +1 and CS +2 both evoked higher proportions of excitatory responses than UCS or CS- (all comparisons $P<0.01$; $\mathrm{CS}+1=91 \% ; \mathrm{CS}+2=90 \%$; UCS $=72 \% ; \mathrm{CS}-=74 \%$, with the remaining responses inhibitory. Most responses tended to be shortlasting $(<2 \mathrm{~s})$, with only a small proportion of responses evoked by $\mathrm{CS}+1$ or CS- persisting for the entire 10 -s duration of the tone $(19 \%$ or $69 / 337$; 60 excitatory, nine inhibitory).

Sensitization increased the proportion of excitatory responses still further compared with non-sensitized rats $(P<0.0001)$ across all tests. Specifically, the CS+2 (sensitized $=95 \%$; non-sensitized $=87 \% ; \quad P<0.01$ ) and UCS (sensitized $=84 \%$; nonsensitized $=60 \% ; P<0.0001)$ both evoked higher proportions of excitatory responses (and fewer inhibitory responses) in sensitized rats than in non-sensitized rats. $\mathrm{CS}+1$ and $\mathrm{CS}-$ proportions were unaffected (both $P>0.05$ ).

The amphetamine challenge did not alter the proportion of excitatory responses compared with vehicle tests, within either sensitized or non-sensitized groups $(P>0.05)$. However, under acute amphetamine challenge there was no longer a difference between sensitized and non-sensitized groups in the proportion of excitatory responses (challenge: sensitized $=86 \%$, non-sensitized $=82 \%, P>$ 0.05 ; vehicle: sensitized $=90 \%$, non-sensitized $=78 \% ; P=0.001$ ).

\section{Normalized firing rates}

The onsets of conditioned stimuli and sucrose UCS triggered changes in firing rates by VP neurons overall, consistent with the firing rate code previously reported (Tindell et al., 2004). The normalized firing rate analysis described here includes all 524 recorded VP units, whether the units had been classified as responsive or non-responsive, excitatory or inhibitory. This ensures that these firing rate codes represent activity of the entire VP population as much as possible.

During all tests, the $\mathrm{CS}+1$ tone and $\mathrm{CS}+2$ click elicited the largest rate increases $(F=26.693, P<0.0001)$, and the magnitude of increase was similar for $\mathrm{CS}+1$ and $\mathrm{CS}+2(P=0.909)$. Both $\mathrm{CS}+1$ and $\mathrm{CS}+2$ rate increases were higher than the UCS rate increase (both $P<0.0001$ ), and higher than the CS- increase (both $P<0.0001$ ). The elevation of $\mathrm{CS}+1$ firing rate over $\mathrm{CS}-$ rate persisted for their entire 10 -s durations (both $P<0.0001$; Fig. 1A). UCS and CS- rates did not differ from each other $(P=0.909)$.

\section{Sensitization enhances neural firing rates for CS+2 and UCS}

Sensitization enhanced increases in firing rates to $\mathrm{CS}+2$ (Fig. $2 \mathrm{~B}$ and C; $F=16.403, P<0.0001)$. Sensitization elevated stimulus-evoked firing overall $(F=22.136, P<0.0001)$, but only $\mathrm{CS}+2$ and its subsequent UCS contributed to this rate elevation (interaction: $F=2.784, P=0.040$; Fig. $2 \mathrm{~B}$ and $\mathrm{C}$ ). There were no detectable increases in firing rates for $\mathrm{CS}+1$ or $\mathrm{CS}-$.

In order to disentangle whether $\mathrm{CS}+2$ firing rates were enhanced the first time sensitized rats encountered them (as predicted by the
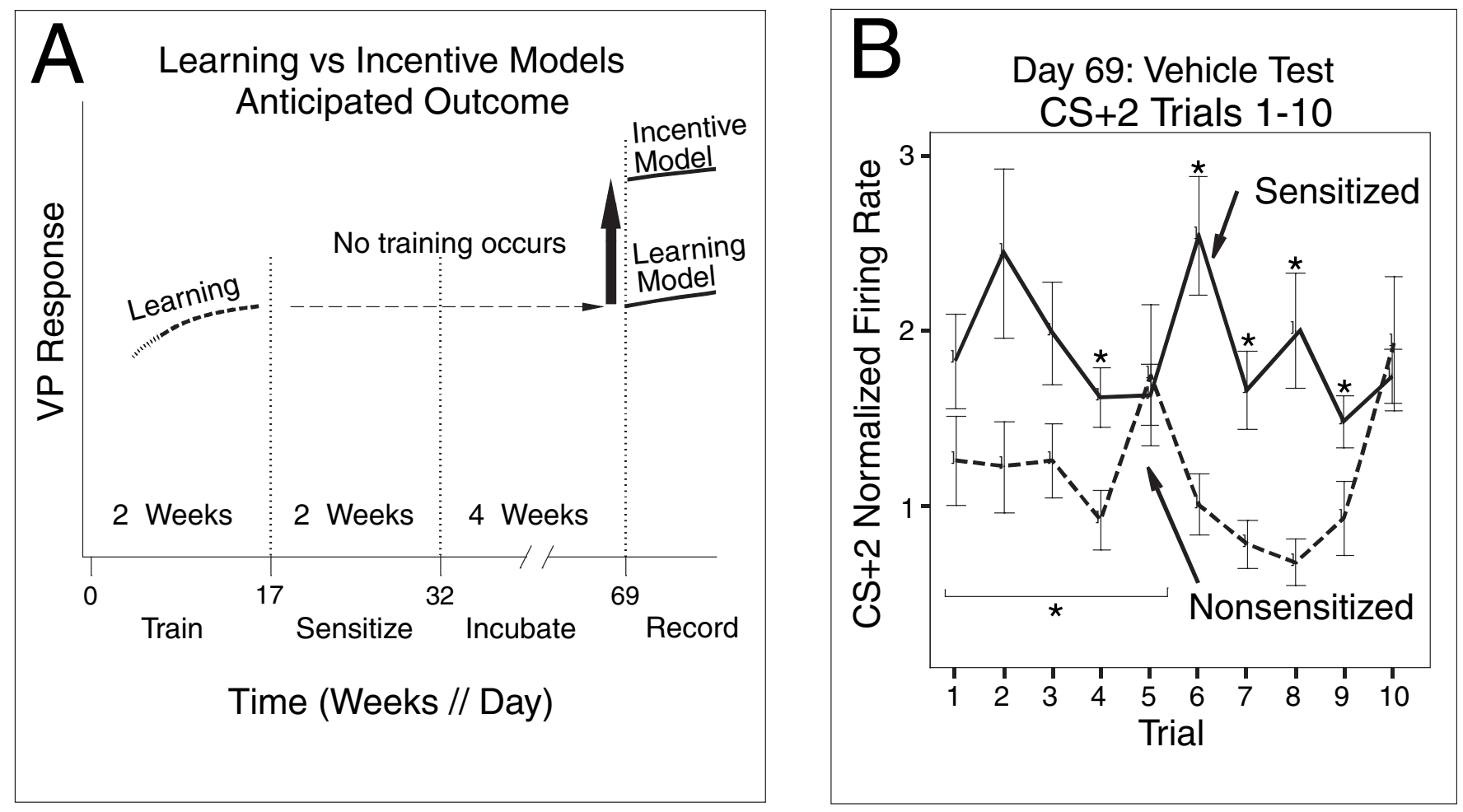

FIG. 3. Incentive sensitization vs. learning sensitization predictions of $\mathrm{CS}+2$ (conditioned stimulus) firing. (A) Hypothetical contrast of data predictions made by incentive vs. learning model for sensitized VP firing to CS+2 on first vehicle test (day 69). Incentive sensitization model predicts immediate firing rate increase to first presentations of CS+2 (double-sided arrow and upper line to right of arrow). By contrast, learning sensitization models predict initial CS+2 firing on day 69 equals level in earlier training (e.g. sensitization of temporal difference error or teaching signal; lower line to right of arrow). Any increase in sensitized learning/signal teaching signal would be gradual and delayed, becoming evident only after additional CS+ and unconditioned stimulus (UCS) pairings (not shown). (B) Increased VP firing to CS +2 is immediately evident on first trials of first vehicle test day for sensitized rats. The first CS +2 trial on the first vehicle test day is already elevated in sensitized animals compared with non-sensitized animals. There is no subsequent growth from the first five trials of the day to the last five trials, further indicating that within-session learning is not contributing to the CS +2 enhancement. ${ }^{*} P<0.05$, comparing groups. 
hypothesis that incentive sensitization increases the attribution of incentive salience to a reward CS+), or instead was only gradually learned after sensitization by subsequent repeated exposures to $\mathrm{CS}+$ and UCS pairings (as predicted by learning-sensitization hypotheses), we specifically examined firing rate changes on the first trials of the first test day. If VP neural coding reflected a prediction learning process, we anticipated delayed and gradual increments in firing that become evident only after further CS-UCS pairings in rats that have been sensitized (Fig. 3A). In contrast, the incentivesensitization hypothesis posits an increase in $\mathrm{CS}+2$ firing rates during the very first trials (Fig. 3A) as a result of sensitizationamplified motivational transformation of previously learned CS+2 signals into increased incentive salience (Wyvell \& Berridge, 2001; Robinson \& Berridge, 2003). Our findings support incentive sensitization: the $\mathrm{CS}+2$ firing rate increase in sensitized rats was visually evident even on the first trial of the first day (sensitized $=180 \%$ vs. non-sensitized control $=120 \%$; Fig. $3 \mathrm{~B}$ ), and reached statistical significance in just the first five trials (the smallest number of trials that allowed statistical comparison; $P=0.026$; Fig. 3B). There was no further increment in firing from the first five trials to the next five trials across the 10 rewarded trials $(F=1.696$, $P=0.113$; Fig. 3B) in either sensitized or non-sensitized animals (no interaction of sensitization with trial: $F=0.998, P=0.453$ ), suggesting that within-session learning was not a significant contributor to the sensitized CS +2 advantage. Overall, the first day increase of $\mathrm{CS}+2$ firing rate in sensitized animals was significant $(P=0.032)$ in comparison to non-sensitized animals. It is noteworthy that this enhancement in $\mathrm{CS}+2$ rates was present even though animals had received no training during the 6 weeks of amphetamine (or control) injections, incubation and surgery, and therefore had no opportunity to learn changes in $\mathrm{CS}+2$ after sensitization based on any associative pairings with UCS. The elevation in firing rates at $\mathrm{CS}+2$ onset in sensitization animals persisted during the period between click and reward $(P=0.009)$ and into UCS onset $(F=5.313, P=0.022$; Fig. 2C). Sensitization did not elevate firing rate to $\mathrm{CS}+1(F=2.160, P=0.142)$ or $\mathrm{CS}-(F=1.753$, $P=0.186$; Fig. 2B).

$\mathrm{CS}+2$ firing rate increases were higher in sensitized than nonsensitized units on each vehicle test (vehicle 1: $250 \%$ vs. $145 \%$, $P=0.032$; vehicle $2: 233 \%$ vs. $161 \%, P=0.002$; washout: $231 \%$ vs. $162 \%, P=0.012$ ), but the amphetamine challenge that also independently raised $\mathrm{CS}+2$ firing in all rats (below) wiped out the statistical significance of the sensitization advantage (315\% vs. $251 \%$, $P=0.200$; see also Fig. 6C). Conversely, the elevation in UCSelicited firing rate caused by sensitization appeared only on the first vehicle test, and was not detectable on any of the other three test days, thus appearing less reliable than the CS+2 elevation (vehicle 1: 141\% vs. $106 \%, P=0.039$; vehicle $2: 154 \%$ vs. $129 \%, P=0.075$; amphetamine challenge: $176 \%$ vs. $153 \%, P=0.303$; washout: $200 \%$ vs. $148 \%, P=0.191)$.

\section{Acute amphetamine amplifies $C S+2$ and $U C S$ rates similar to sensitization}

Amphetamine administration $(2 \mathrm{mg} / \mathrm{kg}$ ) produced $\mathrm{CS}+2$ firing rate increases in all rats similar to those induced by prior sensitization (non-sensitized: $P=0.000$; sensitized: $P=0.041$ ). Normalized firing rates during $\mathrm{CS}+2$ increased during the amphetamine challenge compared with vehicle tests overall $(F=5.447, P=0.001 ; 278 \%$ vs. $192 \%$; Fig. $2 \mathrm{D}$ and E). Again, we observed a jump in $\mathrm{CS}+2$ firing rates on the first trials of the day when amphetamine was administered (comparing the first trial of the amphetamine test with the 10th trial of the immediately preceding day under vehicle administration [groups combined: $P<0.0001$, vehicle: $170 \%$, amphetamine: $280 \%$; sensitized: $P=0.001,180 \%$ vs. $300 \%$; nonsensitized: $P=0.001,150 \%$ vs. $260 \%$ ]) There was no evidence for any gradual increase across trials $1-10$ within the amphetamine day $(P=0.927)$. In other words, amphetamine caused CS +2 firing to start high on the first trial, and to stay at that level across subsequent trials. As with sensitization, the $\mathrm{CS}+2$ rate elevation persisted during the following 1-s stimulus-free period, and into UCS onset ( $P=0.011 ; 163 \%$ vs. $149 \%$; Fig. $2 \mathrm{E})$.

$\mathrm{CS}+2$ and UCS were the only stimuli to have firing rates raised by acute amphetamine (interaction: $F=2.302, P=0.014$; Fig. 2D). Acute amphetamine administration had no effect on firing rates to $\mathrm{CS}+1 \quad(F=0.156, \quad P=0.926$; Fig. $2 \mathrm{D})$ or $\mathrm{CS}-(F=1.002$, $P=0.391$; Fig. 2D) during the four tests. $C S+1$ still elicited faster firing than $\mathrm{CS}-$ in both groups on the amphetamine test, indicating that their Pavlovian discrimination remained intact (sensitized: $P=0.048$; non-sensitized: $P=0.010$ ).

On the fourth day vehicle retest (washout), firing rates to $\mathrm{CS}+2$ declined again from their amphetamine levels on the previous day $(P=0.004)$. On the washout Day 4 , when rats received saline injections, CS+2 firing rates were no longer higher than previous Day 2 vehicle levels $(P=0.376)$. Thus, the magnification of $\mathrm{CS}+2$ firing by systemic amphetamine administration was reversible, and disappeared by the next day.

\section{Baseline firing rate}

Acute amphetamine reduced absolute VP firing rates during the baseline period (vehicle: 11.35 spikes/s; amphetamine challenge: 7.78 spikes/ s; $F=2.865, P=0.036)$. Similar findings have been reported for nucleus accumbens (Peoples et al., 1999; O'Donnell, 2003). In contrast, sensitization had no effect on absolute baseline firing rates $(F=1.088, P=0.354)$.

\section{Firing rates of responsive vs. non-responsive subpopulations}

The firing rate analysis above was highly conservative regarding VP rate coding because it included even non-responsive neurons that did not reach statistical criterion for CS or UCS responsiveness (Fig. 4A and D). The conservative analysis was chosen to be sure that rate codes reflect the entire VP population overall. To assess responsive and non-responsive populations, we also analysed sensitization and acute amphetamine effects on each group separately. Interestingly, for sensitization, the $\mathrm{CS}+2$ enhancement effects described above applied to both the $\mathrm{CS}+2$ responsive population $(F=6.499, P=0.011$; Fig. 4B) and to the non-responsive population of neurons that did reach the activation threshold to be considered $\mathrm{CS}+2$ responsive (perhaps because of variability of firing rates over trials; $F=5.033$, $P=0.027$; Fig. 4A and C). That suggests that even 'non-responsive' neurons carry a moderate $\mathrm{CS}+2$ signal that can be enhanced by sensitization, and that these firing codes reflect firing rates of most VP neurons and not only of a 'responsive' subpopulation. The sensitized firing rate code therefore is to some extent independent of the population code, consistent with our earlier findings (Tindell et al., 2004).

By contrast, after acute amphetamine, the increased normalized firing rate to $\mathrm{CS}+2$ during the four tests was restricted to the responsive subpopulation ( $F=8.279, P<0.0001$; Fig. $4 \mathrm{E})$, with no contribution from the non-responsive subpopulation $(F=1.052$, $P=0.373$; Fig. 4F). Thus, enhancement of firing rates and population representation for $\mathrm{CS}+2$ by acute amphetamine (Fig. 4C) originates from the same subpopulation of responsive 

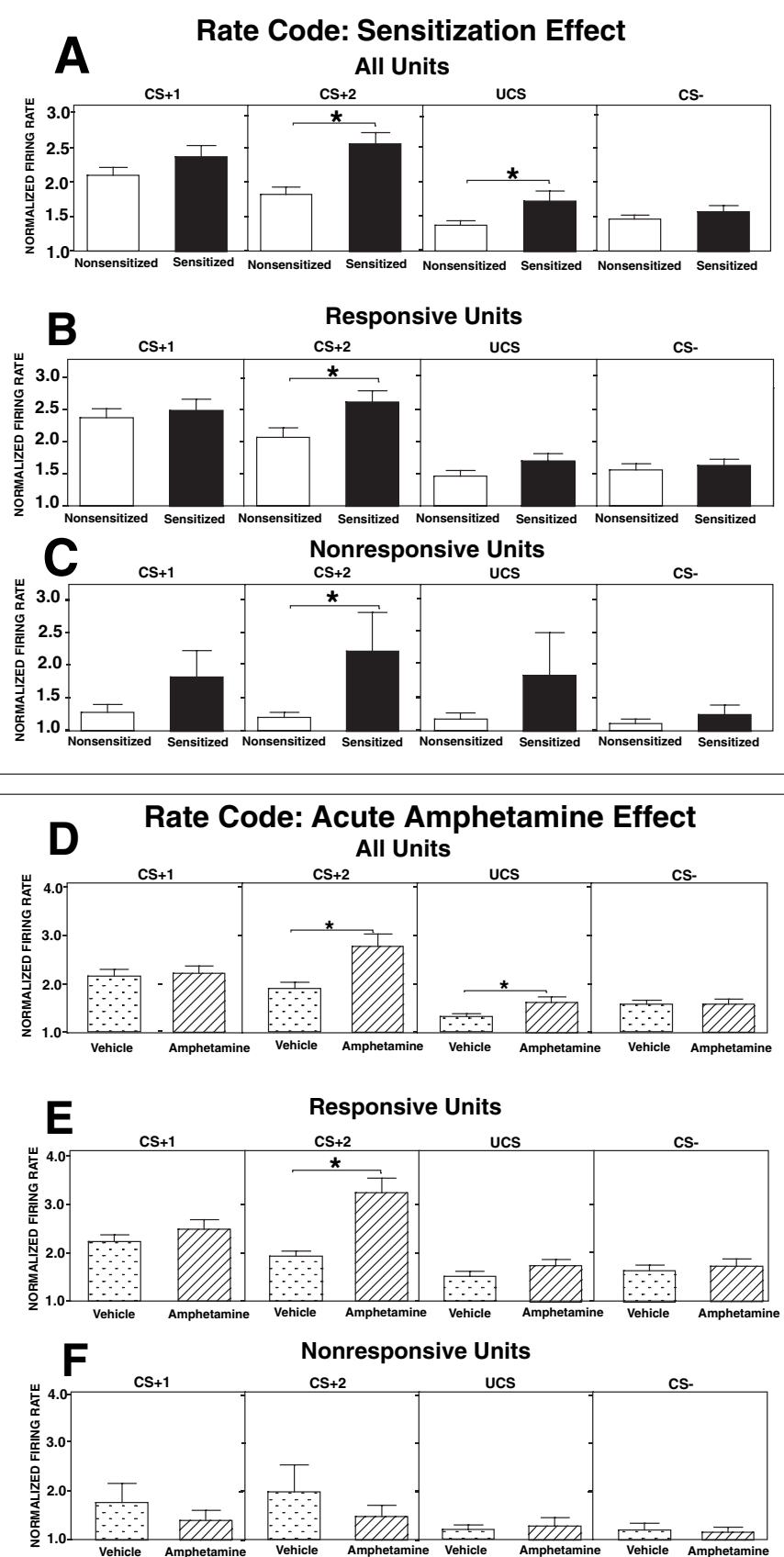

FIG. 4. Responsive and non-responsive VP unit firing rate coding of stimuli during the four tests. (A-C) Sensitization enhancement of CS +2 coding is observed in both 'responsive' units that met criterion for responding to click and 'non-responsive' VP units that did not. (D-F) By contrast, acute amphetamine enhancement of VP rate code for CS +2 comes only from the subpopulations of 'responsive' units that significantly altered firing to that stimulus. No detectable amphetamine contribution comes from the remaining 'non-responsive units'. Thus, amphetamine enhancement to rate coding arises from the same VP neurons that constitute the amphetamine-enhanced population code. $* P<0.05$, comparing groups.

VP units. These results suggest that responsive units may fully explain our amphetamine results, but that even non-responsive populations may also contribute to overall VP firing rates, at least for sensitization effects.

'Profile Analysis': evaluating prediction-coding, value-coding and incentive-coding hypotheses

Most (73\%) VP neurons were activated by multiple stimuli, suggesting a high degree of computational integration within each neuron. To characterize how neurons that respond to more than one stimulus might code differences between stimuli, we employed a new technique called Profile Analysis. We used this Profile Analysis technique to evaluate relative neural activation patterns to $\mathrm{CS}+1, \mathrm{CS}+2$ and UCS with regard to three hypothetical coding schemes. (1) Prediction signal (or TDerror) coding, where the dominant response is to $\mathrm{CS}+1$, the first cue that carries the most predictive information by fully predicting all upcoming stimuli and rewards (Schultz et al., 1997). (2) Incentive motivation coding (incentive salience), where the dominant response is to $\mathrm{CS}+2$, which is most proximal to reward and carries the most 
Pavlovian motivational control over behavior (Corbit \& Balleine, 2003). (3) UCS value coding (e.g. reward hedonic impact, associative stamping-in value or TD value), where the dominant prominent activation is to the UCS, which has most actual reward value.

The profile for each unit is a vector in a two-dimensional 'profile space', a circular scale that represents all possible activation profiles of $\mathrm{CS}+1, \mathrm{CS}+2$ and UCS. The direction of this vector reflects the rankordering of the normalized firing rate responses to $\mathrm{CS}+1, \mathrm{CS}+2$ and UCS, while the magnitude of the vector reflects the extent of the relative differences between the three responses (e.g. the degree to which response to one stimulus dominates the responses to others; Fig. 5A).
Overall, VP neurons had broadly dispersed profiles. The Population Profile vector for the entire VP population (average of all 524 unit profile vectors, Fig. 5A) lies between prediction- and incentivedominant regions of profile space. The distribution of profiles (counts of all 524 units in $30^{\circ}$ bins across profile space, Fig. 5A) reinforces the broadly dispersed nature of firing profiles between prediction signalcoding and incentive-coding regions with only a small contribution from value-coding profiles.

We computed Population Profile Vectors and distributions of profiles to test the effects of sensitization and direct amphetamine challenge. Both sensitization (Fig. 5B) and acute amphetamine
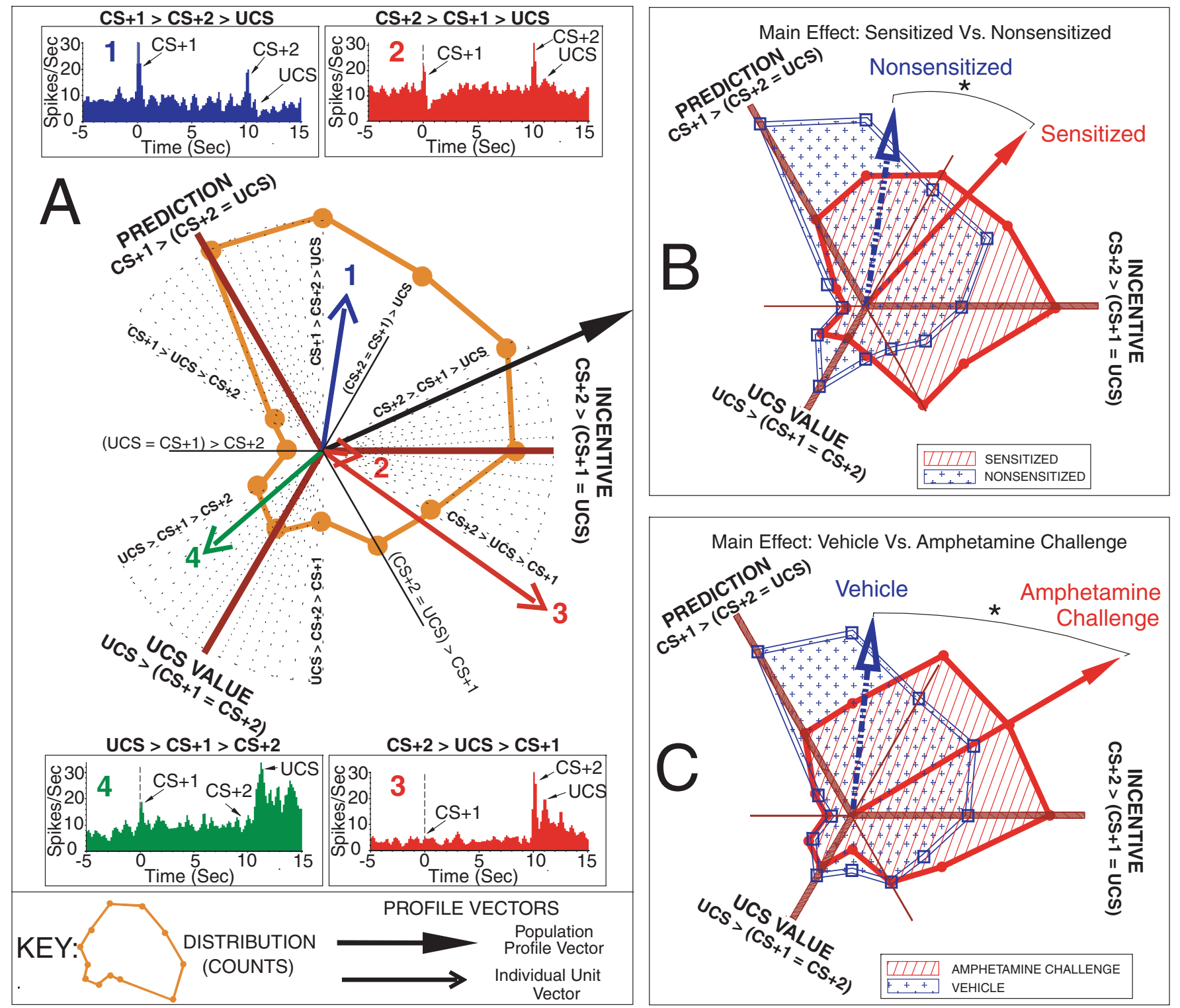

FIG. 5. Profile analysis. (A) Distribution of all 524 VP neuronal populations' firing responses to CS +1 , CS+2 and sucrose unconditioned stimulus (UCS) during the four tests is shown as a frequency histogram (orange dots and lines). The black arrow indicates the Population Profile Vector for all recorded VP neurons. The four inset histograms at top and bottom and their corresponding unit vectors (arrows 1-4) show examples of individual neural units coding a prediction profile (CS+1 bias; 1 blue histogram and vector arrow), incentive profiles (CS +2 bias; 2, 3 red histograms and arrows), or hedonic value profile (UCS bias; 4 green histogram and arrow). (B) Sensitization shift of profiles from prediction coding to incentive coding. The non-sensitized population (blue; right dotted hatching) is biased toward predictive coding (while also extending to incentive-coding regions), while the sensitized population is biased toward incentive coding (red; slant hatching; main effect of sensitization includes both vehicle and amphetamine test days). The Population Profile Vector arrows show a corresponding shift in maximal response towards CS+2 'incentive-coding' region. Axes have been trimmed for graphical presentation. (C) Acute amphetamine (test 3) similarly shifts VP neurons toward incentive-coding profiles. Amphetamine shifts the distribution of responses and the Population Profile Vector away from the prediction-coding sector that dominated during vehicle tests (tests 1,2 and 4) and towards the incentive-coding sector (main effect of amphetamine includes both sensitized and non-sensitized firing). $* P<0.05$, asterisks between vector arrows denote a significant shift in vector direction. 
(Fig. 5C) shifted the distributions of response profiles in similar fashions: away from prediction signal coding (neurons with strongest firing to $\mathrm{CS}+1$ ) toward incentive coding (neurons with strongest firing to $\mathrm{CS}+2$; hatched areas Fig. $6 \mathrm{~A}-\mathrm{D}$ ). The additive combination of amphetamine administered to already sensitized rats produced the strongest profile shift, resulting in a nearly pure incentive-coding profile (Fig. 7). Thus, mesolimbic activation had a potent effect on population distributions of VP neuronal profiles.

The shift of the Population Profile Vector toward incentive-coding $(\mathrm{CS}+2)$ profiles by sensitization was significant $(P=0.012$, MANOVA; vector Fig. 5B). The amphetamine challenge also produced a significant shift of profiles in the same direction toward incentive- coding regions of the profile space $(P<0.0001$, MANOVA; vector Fig. 5C). Overall, the effect of the mesolimbic activation across groups can be visualized as a rotation of the Population Profile Vectors and distributions across profile space (Fig. 6). With increasing intensities of mesolimbic activation, this rotation transforms the profiles from predictive coding in normal animals in vehicle test toward more incentive coding regions of profile space (Fig. 7). Intermediate shifts appear in sensitized animals receiving vehicle as well as in nonsensitized animals receiving amphetamine (Fig. 7). In individual comparisons, all between-group differences were significant, except comparing sensitized and non-sensitized animals under amphetamine (MANOVA: non-sensitized + vehicle vs. sensitized + vehicle, $P=$

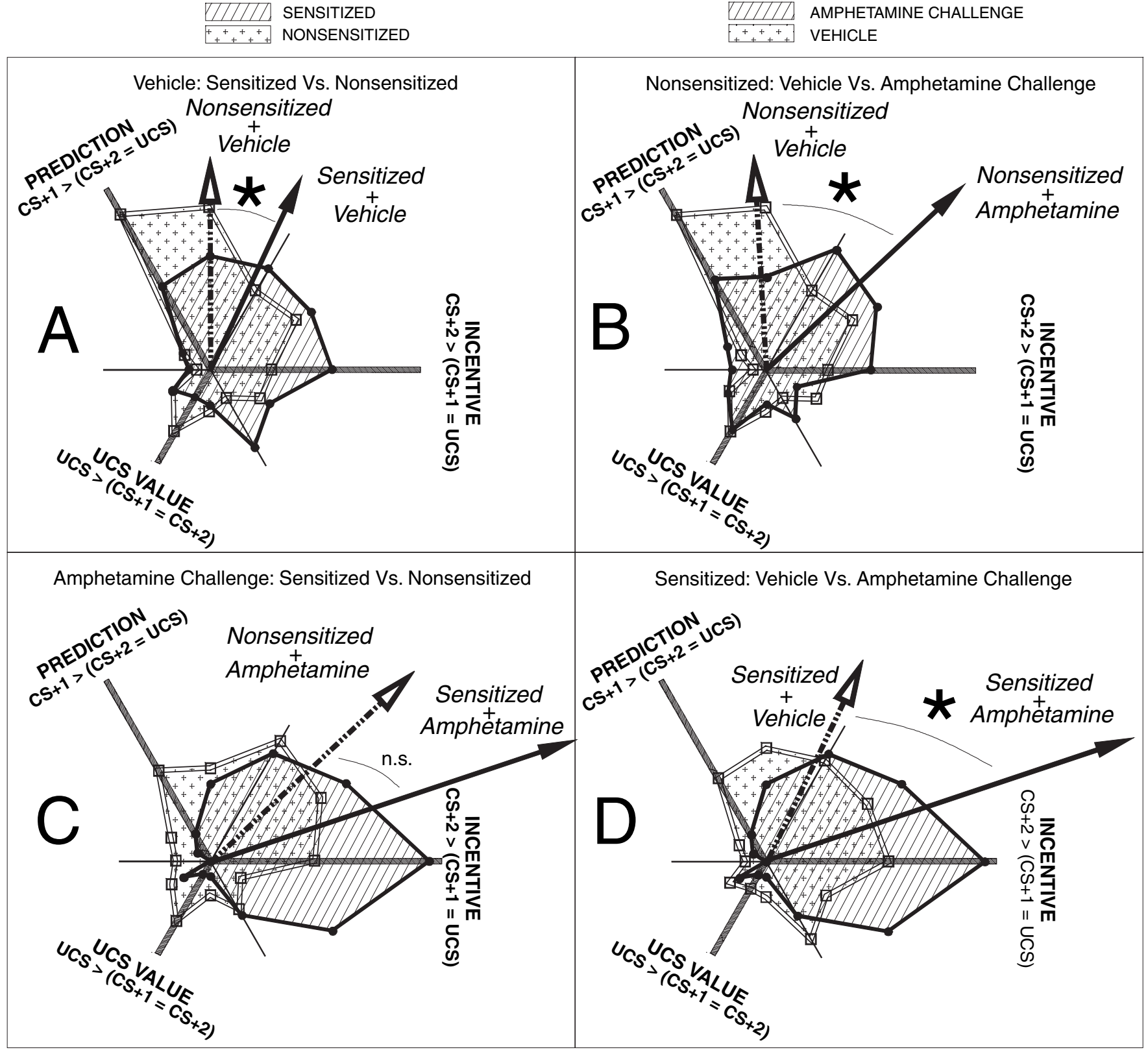

FIG. 6. Profile analytic breakdown of non-sensitized vehicle, sensitized vehicle, non-sensitized amphetamine and sensitized amphetamine conditions. (A) Nonsensitized vehicle profiles show a strong preference toward prediction signal coding $(\mathrm{CS}+1)$, but the profiles for the sensitized population are shifted toward incentive coding (CS+2), when both groups of rats are tested under vehicle (first test days). (B) Acute amphetamine shifts non-sensitized VP firing incentive coding $(\mathrm{CS}+2)$ compared with non-sensitized vehicle tests. (C) Amphetamine causes a marginally greater incentive shift in VP firing profiles in sensitized rats than in nonsensitized rats. (D) Acute amphetamine shifts sensitized VP firing even further toward incentive coding (CS+2) compared with VP vehicle profiles measured in the same sensitized rats. ${ }^{*} P<0.05$, asterisks between vector arrows denote a significant shift in vector direction. 


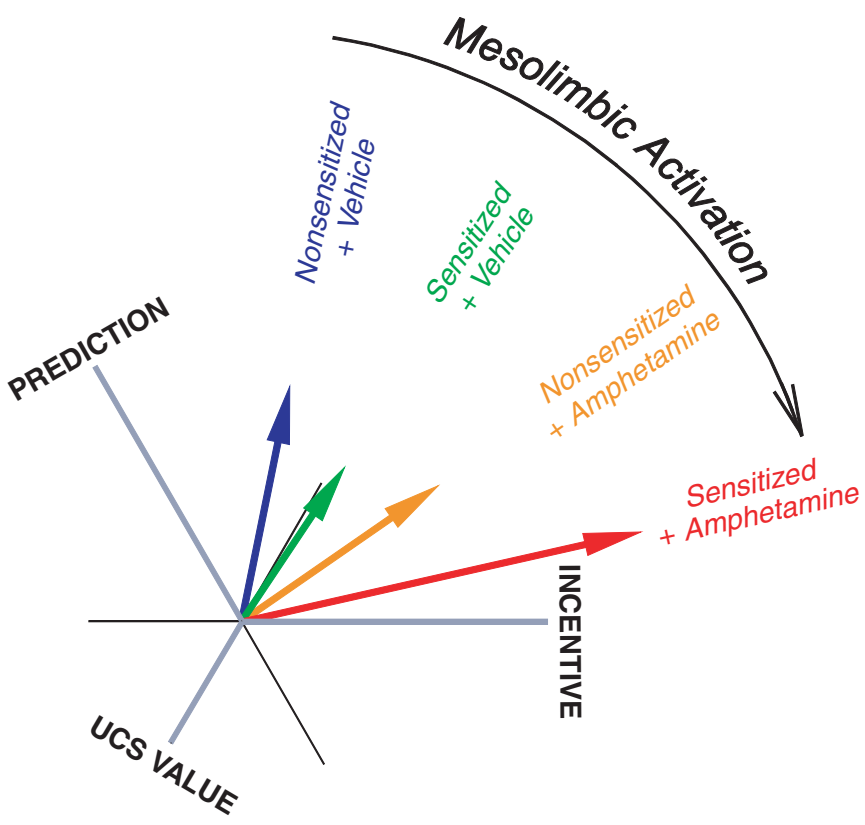

FIG. 7. Population Profile Vector shifts toward incentive coding with mesolimbic activation. The Population Profile Vector for all recorded neurons in each group of animals is shown for comparison. VP firing in non-sensitized animals during vehicle tests (tests 1,2 and 4) reveals a profile closest to that of prediction coding ( $\mathrm{CS}+1$ bias), while firing in sensitized animals during amphetamine challenge (test 3 ) reveals a profile closest to that of incentive coding ( $\mathrm{CS}+2$ bias).

0.011; non-sensitized + vehicle vs. non-sensitized + amphetamine, $P<0.0001$; non-sensitized + amphetamine vs. sensitized + amphetamine, $P=0.339$; sensitized + vehicle vs. sensitized + amphetamine,
$P=0.001$; Figs 6 and 7). Thus, VP neurons in normal animals tend to follow a prediction signal-coding profile, but with mesolimbic activation by either sensitization or acute amphetamine administration, VP neurons increasingly shift towards encoding incentive salience in a potentially additive fashion (Fig. 7).

\section{Movement controls}

Most VP units that responded to reward CS or UCS stimuli did not appear to be triggered by identifiable movements. For example, among units that responded to $\mathrm{CS}+1$, only $2.8 \%$ (4 units) showed neural activity correlated with a turn of the head, and only $7.8 \%$ (11 units) showed activation related to a stepping movement emitted during that stimulus (Fig. 8). In the CS- responsive population, 6\% (6 units) showed activation with head movements, and $7.8 \%$ ( 8 units) changed activity for stepping movements that occurred during the CSpresentation (Fig. 8). Likewise in the UCS-responsive population, we found $6 \%$ (5 units) that showed activity correlated with oral movements (licking) during body grooming outside the context of UCS consumption (Fig. 8). We also examined movements during the intertrial period, when no stimulus was present. Percentages of units with neural activity correlated to specific movements during intertrial periods were at similarly low levels for each movement category (all 6-7\%). These few movement responses were equally distributed in sensitized and non-sensitized rats, and in vehicle and amphetamine conditions. Therefore a motor or movement-coding hypothesis for VP firing does not appear to account for our CS or UCS results (Fig. 8).

\section{Behavioral responses}

Locomotion and stereotypy. Sensitization was confirmed by higher locomotion in sensitized rats than non-sensitized rats overall (sensitization effect: $F=18.357, P<0.0001$; Fig. 9A). Amphetamine

\section{STIMULUS EVENT}

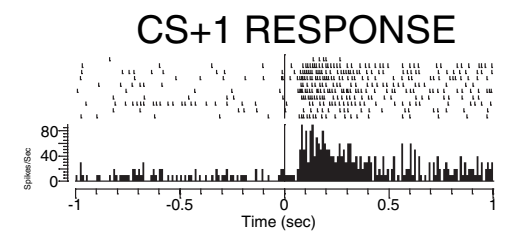

\section{CS- RESPONSE}

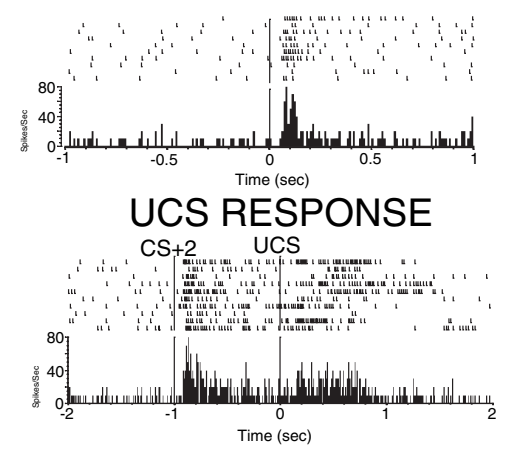

\section{MOTOR CONTROL EVENT}
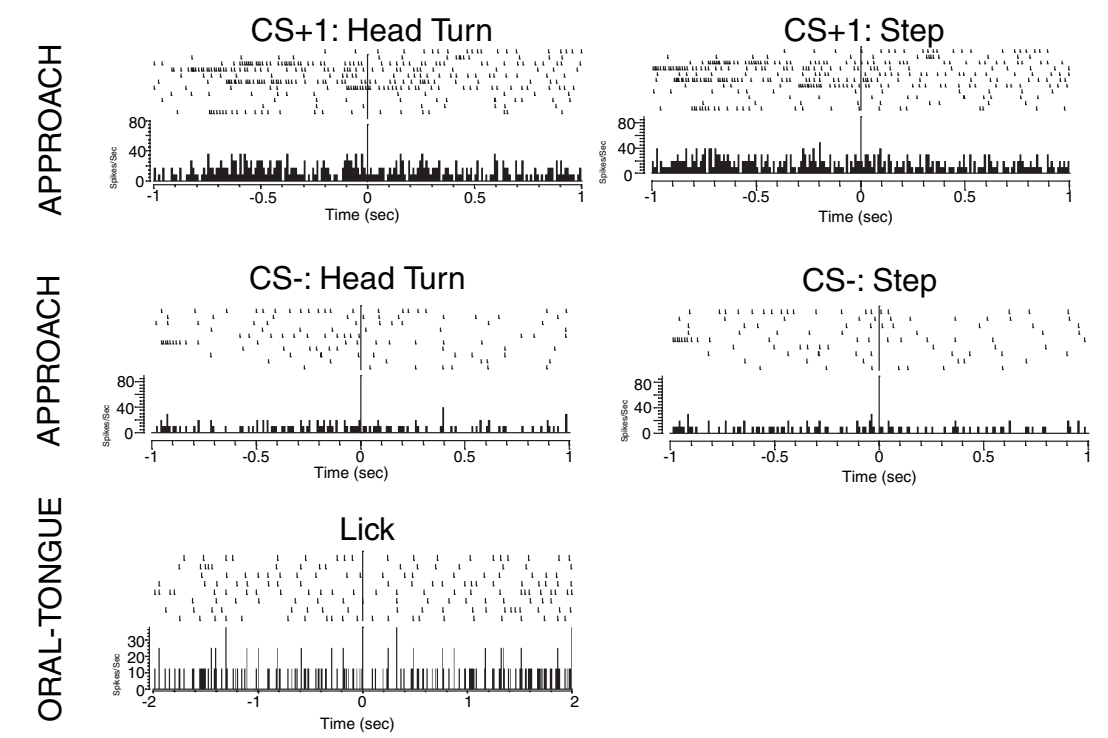

FIG. 8. VP neurons that fire conditioned stimulus (CS) and unconditioned stimulus (UCS) do not code concurrent movements. Example of one VP unit that responded to Pavlovian reward stimuli (left). Examples of firing from one VP neuron that responded to CS+1, CS- and UCS (left). The same VP unit fails to fire during head movements or stepping movements occurring during those CS tones, or to mouth and tongue movements that occurred spontaneously in grooming contexts. 
increased stereotypy scores across all rats (test effect: $F=25.665$, $P<0.0001$; Fig. 9A). Sensitized rats showed markedly higher stereotypy than non-sensitized rats on the day of amphetamine administration (sensitized stereotypy score $=5.47$; non-sensitized $=3.89 ; P<0.0001)$. Higher stereotypy showed that psychomotor sensitization had indeed been produced by the earlier sensitizing 2 -week drug regimen they had received a month earlier.

Conditioned approaches. Behavioral conditioned approach responses confirmed that all rats had successfully formed discriminative associations regarding $\mathrm{CS}+$ vs. $\mathrm{CS}-$ relations to reward. Rats were generally away from the sucrose dish at tone onsets, either resting or exploring the chamber. Most rats responded immediately at $\mathrm{CS}+1$ tone onset with a head turn or step toward the sucrose dish (in approximately $2 \mathrm{~s}$ ), and it took them $4 \mathrm{~s}$ on average to make a first nose poke at the sucrose dish. Typically once at the sucrose dish midway through $\mathrm{CS}+1$ tone, they remained there until $\mathrm{CS}+2$, and became increasingly more likely to make nose pokes as $\mathrm{CS}+2$ and UCS approached. Rats were less likely to approach the sucrose dish during the $\mathrm{CS}-$ than the $\mathrm{CS}+(\mathrm{CS}+1: 4.43 \pm 0.48$ nose pokes; $\mathrm{CS}-$ : $0.49 \pm 0.12 ; F=63.148, P<0.0001$; Fig. 9B), they were slower to do so, and did not remain at the sucrose bowl for the duration of the tone. There were no differences between sensitized and non-sensitized rats or between vehicle and amphetamine sessions in approach behaviors, including latency to respond to tones, duration of nose pokes or the duration of time rats were in contact with the sucrose bowl (e.g. paw on sucrose bowl).

Sensitized and non-sensitized rats did not differ in the total number of nose pokes to the sucrose bowl (no sensitization effect: $F=1.109$,
$P=0.294)$. This similarity between sensitized and non-sensitized conditioned approaches mitigates motor explanations for sensitization enhancement of CS+2 VP firing. We know of no reports that sensitization induced after learning increases conditioned approach responses, suggesting that conditioned approach becomes a relatively constant motor habit with training.

Acute amphetamine actually suppressed nose pokes compared with vehicle tests (test effect: $F=4.071, P<0.008$; Fig. 9B). The behavioral stereotypies caused by amphetamine possibly may have competed with approach responses and suppressed them (e.g. stereotyped head and mouth movements; above). Amphetamine specifically reduced the high level of dish nose pokes elicited by the $\mathrm{CS}+1$, while the already lower CS- approach level was not affected by amphetamine (interaction: $F=3.871, P<0.001$ ). In any case, amphetamine suppression of dish approach again indicates that amphetamine enhancement of VP coding of $\mathrm{CS}+2$ cannot be explained by motor aspects of an enhanced behavioral conditioned response.

\section{Sensitization/amphetamine suppression of behavioral taste 'liking' and 'disliking' reactions (Experiment 2)}

Given that enhanced VP firing rates triggered by CS +2 often persisted into sucrose UCS on the first test day (described above), it seemed crucial to independently assess whether sensitization or acute amphetamine increased the hedonic reward impact ('liking') of unconditioned tastes (even though the profile analysis above indicated UCS value was not enhanced). One useful and independent measure
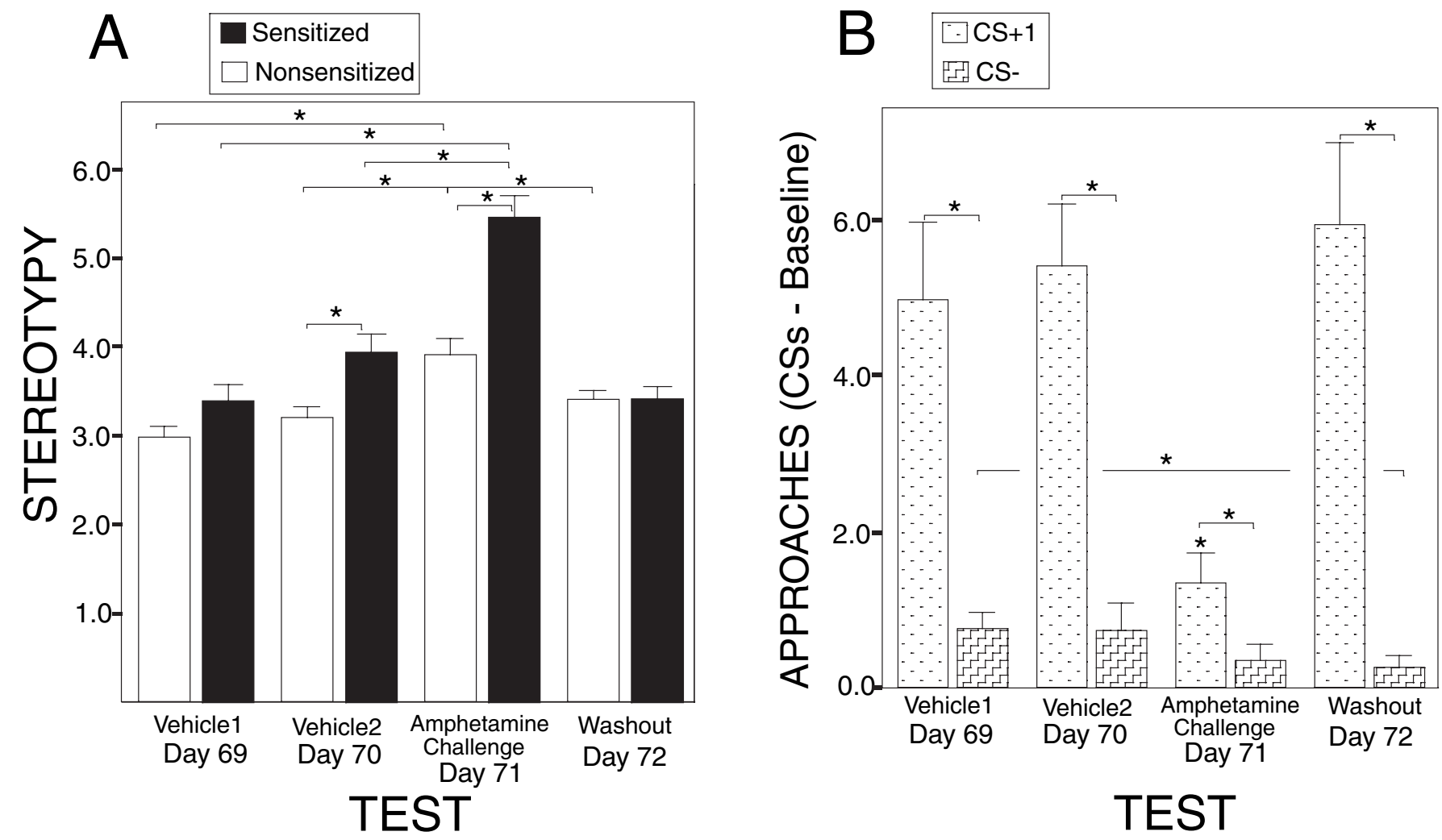

FIG. 9. Behavioral stereotypy and conditioned approaches. (A) Locomotion and stereotypy scores were higher in sensitized rats than in non-sensitized rats, especially on the acute amphetamine test day. This pattern confirmed that sensitization had been induced by the prior drug regimen. (B) Behavioral conditioned approaches to the sucrose dish elicited by the tone CS +1 (compared with pre-CS baseline) on each day. The CS +1 tone potently elicited conditioned approach overall, though behavioral approaches were reduced on the day of acute amphetamine administration. $* P<0.05$, comparing bracketed groups. 


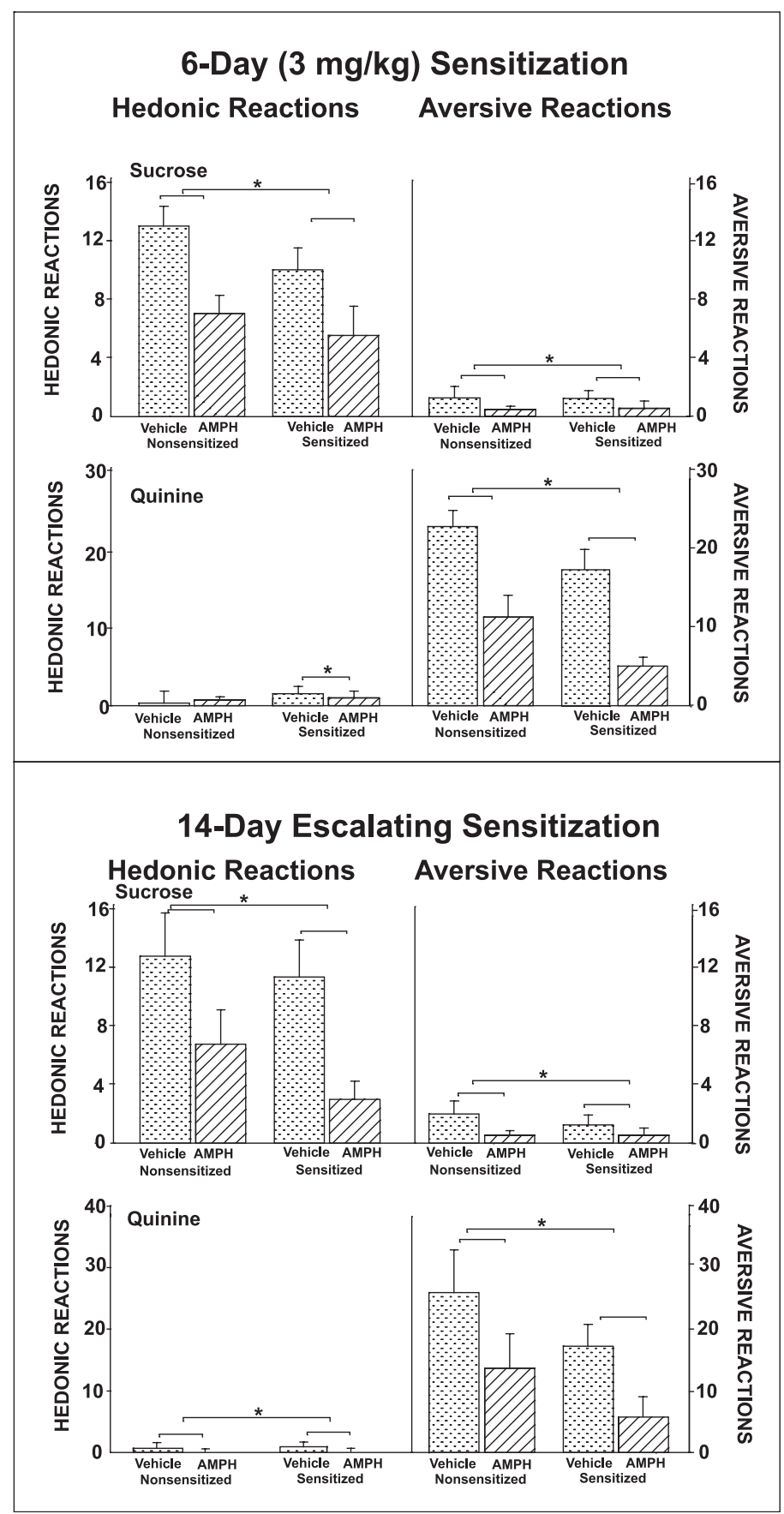

FIG. 10. Sensitization fails to increase hedonic 'liking' for UCS, measured by behavioral taste reactivity to sucrose. Sensitized rats in both the 6-day (top panel) and the 14-day escalation (bottom panel) groups showed decreased affective reactions to both sucrose and quinine tastes compared with non-sensitized rats. Amphetamine administration also decreased 'liking' reactions to sucrose and 'disliking' reactions to quinine. Thus, sensitization and amphetamine each fail to increase taste hedonic impact or 'liking' reactions. Instead sensitization/amphetamine forms of mesolimbic activation only appear to blunt all affective reactions regardless of positive/negative valence. Error bars represent mean \pm SEM hedonic or aversive affective reactions. ${ }^{*} P<0.05$, comparing bracketed groups.

of sucrose hedonic impact is behavioral affective taste reactivity patterns, or orofacial 'liking' reactions homologous to human affective facial expressions. Thus, 28 additional rats were implanted with oral cannulae and tested in a taste reactivity paradigm to assess sucrose hedonic 'liking', 1 month after an amphetamine sensitization regimen (either 6 days; Wyvell \& Berridge, 2001; or 14 days as in Experiment 1) or a non-sensitizing vehicle regimen. Affective reactions to infusions of sucrose or quinine into the mouth were recorded after amphetamine or vehicle administration, and behavioral orofacial 'liking' and 'disliking' reactions were scored in a slow-motion video analysis (Berridge, 2000). We found that sensitization/amphetamine only suppressed taste reactivity 'liking' and 'disliking' expressions elicited by sucrose or quinine tastes, and never increased positive 'liking' expressions (Fig. 10).

Affective 'liking' reactions to sucrose and 'disliking' reactions to quinine were both suppressed overall by prior sensitization compared with non-sensitized control rats [6-day microinjection sensitization group: $F=37.96, P<0.001$; Fig. 10 (top); 14-day group: $F=5.12$, $P<0.05]$. Similarly, acute amphetamine microinjection on the day of test also suppressed both positive and negative affective reactions to 
sucrose and quinine in both sensitized and non-sensitized rats ( $F=8.15-14.99, P<0.01$ for all groups; Fig. 10$)$.

In summary, mesolimbic activation suppressed affective 'liking' and 'disliking' reactions to tastes, and never increased positive 'liking' reactions to sucrose. Thus, neither sensitization nor acute amphetamine administration increased the hedonic impact of sucrose taste, providing further evidence against UCS value magnification.

\section{Discussion}

Our analysis of VP coding revealed important new findings about reward stimulus processing by VP neurons. First, we found that VP neural activity integrates predictive, incentive and reward value information in the sense that most VP neurons respond to more than one reward stimulus $(\mathrm{CS}+1, \mathrm{CS}+2, \mathrm{UCS})$ and thus encode more than one of those reward features. Our profile analysis showed that predictive coding (i.e. CS+1) ordinarily dominates in normal rats that have learned the Pavlovian prediction. Second, we found that activating mesolimbic circuits by either sensitization or acute amphetamine incrementally causes a specific VP coding shift away from relative prediction coding and towards stronger relative incentive coding (i.e. $\mathrm{CS}+2$ ). That sensitization/amphetamine shift to an incentive bias for CS+ coding occurred as soon as $\mathrm{CS}+2$ was encountered in the activated mesolimbic state, and did not need to be relearned in that new state. It appeared on the very first trials of the first relevant test day when there had yet been no opportunity for relearning (Fig. 3B). Sensitization and amphetamineinduced incentive shifts appeared to be additive, in the sense that the incentive coding of sensitized rats was even greater when they also received acute amphetamine.

These shifts toward VP incentive coding were not due to enhanced UCS hedonic impact ('liking'), as behavioral hedonic 'liking' reactions to sucrose taste remained constant or even diminished slightly with sensitization and amphetamine administration. These findings are consistent with a specific role for VP neurons in attributing incentive salience to reward-related stimuli based on an integration of previously learned associations together with current mesolimbic activation. They also suggest how sensitization and addictive drugs may prime motivational behavioral responses of addicts to drug-related stimuli by amplifying the incentive impact of encountering a UCS-proximal drug $\mathrm{CS}+$. Finally, they suggest an incentive salience mechanism to help explain why temporal discounting reduces the incentive impact more for an early $\mathrm{CS}+$ that is temporally distant from its reward than for a later $\mathrm{CS}+$ that is temporally proximal to its UCS: the UCS-proximal CS+2 is attributed with greater incentive salience by VP circuits, especially in states of mesolimbic activation.

\section{Mesolimbic activation causes shift to $C S+2$ away from $C S+1$}

The shift toward incentive coding was shown by stronger firing in more VP neurons, specifically to the second conditioned stimulus (CS+2 feeder click) that was temporally closest to the actual reward (UCS). Our analysis of neural firing rate response profiles showed that VP neural populations shifted their response preference away from the most predictive conditioned stimulus $(\mathrm{CS}+1)$, which dominated in control rats, toward the stronger incentive stimulus $(\mathrm{CS}+2)$, which dominated in sensitized rats and in all rats when tested under acute amphetamine (Fig. 5). Finally, the acute amphetamine-induced shift toward incentive coding disappeared the next day under vehicle retest, indicating that the coding change was reversible and pharmacologically state dependent. These complex and specific changes in VP coding may arise from the complex network of signals VP neurons receive from other mesocorticolimbic structures, including $\gamma$-aminobutyric acid (GABA) and opioids from accumbens (Usuda et al., 1998; Tang et al., 2005), glutamate from amygdala and other forebrain structures (Galaverna et al., 1993; Turner et al., 2001), and dopamine from the ventral tegmental area (Kalivas \& Nakamura, 1999).

It is well recognized that the second $\mathrm{CS}+2$ in a series of conditioned stimuli carries less predictive information than an earlier CS+1 that predicts both it and reward UCS (Schultz et al., 1997). Yet the second stimulus in a series may still carry more incentive salience value. As Corbit and Balleine note, '...the proximal [second] action is associated with the most salient feature of the outcome that is itself directly associated with motivational structures that mediate the biological significance of the outcome' (Corbit \& Balleine, 2003, p. 105). Such considerations suggest that our CS+2 may carry greater motivational impact than $\mathrm{CS}+1$, because $\mathrm{CS}+2$ is closer in time to UCS (Giordano et al., 2002; Corbit \& Balleine, 2003). In part, this may be a mechanism for causing temporal discounting, the phenomenon in which the motivating impact of rewards and reward-related stimuli weakens disproportionately as they become temporally more distant (Vuchinich \& Simpson, 1998; Critchfield \& Kollins, 2001). In the present study, the CS +2 signal appeared at a moment of strong incentive anticipation, reflected behaviorally in rats' more frequent nose pokes into the sucrose dish around the moment of $\mathrm{CS}+2$ than during the earlier first half of $\mathrm{CS}+1$. These considerations suggest that incentive motivation may grow in parallel with the temporal pattern of VP incentive coding we observed. Mesolimbic activation appeared to shift the VP bias from predictive coding to incentive coding, although CS predictive and UCS value coding continued to be integrated to lesser degrees by the same neurons.

Integrative coding of both $\mathrm{CS}+1$ and $\mathrm{CS}+2$ is in keeping with previous work on VP coding of serial $\mathrm{CS}+\mathrm{s}$ during training (Tindell et al., 2004). However, previously we found that the CS+2 population size dropped from early to later learning trials (Tindell et al., 2004). The observation here that a robust $\mathrm{CS}+2$ population remained on test trials may be due to several procedural differences between our present experiment on $\mathrm{CS}+$ recall and our earlier experiment on $\mathrm{CS}+$ acquisition (including a 6-week time-off period for sensitization and incubation between training and test here, which may have allowed partial forgetting, as well as a greater number of training trials here). In any case, integrative coding of $\mathrm{CS}+1, \mathrm{CS}+2$ and UCS appears to characterize VP firing during reward learning acquisition and learning recall, but their balance of coding appears to shift with the course of learning and with mesolimbic activation.

\section{A learning explanation?}

A pure learning explanation of our VP incentive results deserves consideration, but it can be ruled out on several grounds. For example, enhanced firing rate responses to $\mathrm{CS}+2$ after sensitization occurred during the first presentations of $\mathrm{CS}+2$, before there was any chance for enhanced relearning about its relation to UCS. Even on the very first trial of the first test day, 6 weeks after training ceased, sensitized rats showed a $50 \%$ greater increase in CS +2 firing than non-sensitized rats. Furthermore, there was no apparent growth of incentive coding across trials during this first test session, indicating that within-session learning was not driving the $\mathrm{CS}+2$ enhancement. Thus, sensitization of incentive coding appears full-blown in magnitude on the first test presentation of CS+2 encountered in the sensitized state, and does not need any relearning after sensitization. Rats received no additional training that could enhance $\mathrm{CS}+2$ associations during their 6-week 
break between sensitization and test. If the shift toward incentive coding with mesolimbic activation was dependent on relearning a stronger Pavlovian association or reward prediction, further pairings of $\mathrm{CS}+$ and UCS after sensitization would have been required (Berke \& Hyman, 2000; Hyman \& Malenka, 2001; Dayan \& Balleine, 2002; Di Chiara, 2002; Everitt \& Wolf, 2002; Montague \& Berns, 2002; Schultz, 2002; Corbit \& Balleine, 2003; Ghitza et al., 2003; Kelley et al., 2003; O'Doherty et al., 2003; Nicola et al., 2004; Redish, 2004). A gradual increase in CS +2 firing due to a relearning process is clearly different from the increase we observed on the very first trial (Fig. 3B).

Similarly, for acute amphetamine administration, enhanced incentive coding of $\mathrm{CS}+2$ occurred full-blown on the first trial of amphetamine challenge for all rats. Enhanced incentive coding did not need relearning trials under amphetamine, and did not grow or benefit from further relearning across trials within the amphetamine day. Finally, amphetamine enhancement of CS+2 incentive coding disappeared the following day in the 'washout' vehicle retest, whereas learning should have remained stable once established, and so amphetamine reversibility further argues against a learning-based interpretation.

\section{An incentive salience explanation}

A better explanation for post-learning increases in VP firing may be the incentive salience interpretation. Mesolimbic activation transforms the learned CS+ signal and imparts an amplified CS+ motivational value to $\mathrm{CS}+$, i.e. conditioned incentive salience. This motivational transformation of a neural $\mathrm{CS}+$ signal depends on integrating two separate factors: (1) current physiological/neurobiological state; (2) previously learned associations about $\mathrm{CS}+$ (Toates, 1986; Berridge \& Robinson, 1998; Dickinson \& Balleine, 2002; Schultz, 2002). Integrating current physiological state with learned cues allows behavior to be guided dynamically by appetiteappropriate stimuli without need of further learning (e.g. Pavlovian cues associated with food are immediately more attractive to a hungry animal). Drug sensitization or acute amphetamine may each 'short circuit' this neurobiological system and directly increase the incentive value attributed to particular conditioned stimuli, triggering greater 'wanting' and pursuit of their reward (Wyvell \& Berridge, 2001; Marinelli et al., 2003; Robinson \& Berridge, 2003).

\section{Do sensitization and acute amphetamine change VP coding of sucrose UCS?}

Amphetamine diminished hedonic taste reactivity responses to tastes, which effectively blunted behavioral affective reactions, an effect almost opposite to hedonic enhancement. In VP, UCS firing rate responses were generally smaller than changes induced by $\mathrm{CS}+1$ and $\mathrm{CS}+2$. Indeed, changes in UCS firing rates might be due in part to persistent activation carried over from $\mathrm{CS}+2$ that occurred only $1 \mathrm{~s}$ earlier (Fig. 2C and E). Our Profile Analysis showed no change in VP coding of UCS with amphetamine. These data suggest that if sensitization or amphetamine increases any aspect of UCS processing, it must be a non-hedonic aspect separate from 'liking' or reward value.

If non-hedonic UCS increments are real, one possible role is dopamine-modulated 'reboosting' of incentive salience. Reboosting is posited to be a trial-by-trial restoration of magnified incentive value attributed to the memory representation of conditioned stimuli that occurs when expected reward is actually obtained (Berridge \&
Valenstein, 1991; Berridge \& Robinson, 1998; Robinson et al., 2005). For example, McClure et al. (2003) suggested that incentive salience reboosting at UCS increments incentive value expressed over subsequent learning trials. Clearly, more work is needed to clarify possible roles of reboosting of UCS, and to disentangle mesolimbic activation effects on UCS vs. CS coding.

\section{General conclusions}

We found that VP neurons integrate prediction and incentive motivation coding of CS+ signals, and that sensitization and amphetamine manipulations shift the neural profiles away from prediction coding and toward motivational (incentive salience) coding. Mesolimbic activation shifts VP computations in a manner that appears to specifically amplify incentive salience coding. Thus, mesolimbic activation may enhance the decision utility of the CS+2 (ability to motivate choice), relative to its predicted utility (expectation of outcome).

Finally, the shift toward a $\mathrm{CS}+2$ coding bias (relative to $\mathrm{CS}+1$ ) suggests that cues that are closest in time to reward gain the most motivational enhancement by mesolimbic activation. This suggests that human drug addicts might be especially vulnerable to drug cues temporally close to drug delivery because sensitized mesolimbic systems involving VP attribute those cues with magnified incentive value, resulting in excessive cue-triggered 'wanting'. Better understanding of these neural computations may clarify not only what VP contributes to mesocorticolimbic circuits for normal motivational function, but also what goes wrong on a moment-by-moment basis in addiction and related disorders of desire.

\section{Acknowledgements}

The authors would like to acknowledge the technical assistance of Nadia Siddiqui, Kyle Smith, Rick Kindt and Ken Marriott, and helpful comments on the manuscript by Wolfram Schultz. The research was supported by NIH, NIDA and NIMH grants NS31650, DA017752, DA015188 and MH63649 (J.W.A. and K.C.B.), and a National Science Foundation Fellowship granted to A.J.T.

\section{Abbreviations}

$\mathrm{CS}+1$, first (or 'distal') conditioned stimulus in a series; CS +2 , second (or 'proximal') conditioned stimulus in a series; UCS, unconditioned stimulus; VP, ventral pallidum.

\section{References}

Aldridge, J.W. \& Berridge, K.C. (1998) Coding of serial order by neostriatal neurons: a 'natural action' approach to movement sequence. J. Neurosci., 18, 2777-2787.

Balleine, B.W., Garner, C., Gonzalez, F. \& Dickinson, A. (1995) Motivational control of heterogeneous instrumental chains. J. Exp. Psych.: Anim. Behav. Proc., 21, 203-217.

Berke, J.D. \& Hyman, S.E. (2000) Addiction, dopamine, and the molecular mechanisms of memory. Neuron, 25, 515-532.

Berridge, K.C. (2000) Measuring hedonic impact in animals and infants: microstructure of affective taste reactivity patterns. Neurosci. Biobehav. Rev., 24, 173-198.

Berridge, K.C. \& Robinson, T.E. (1998) What is the role of dopamine in reward: hedonic impact, reward learning, or incentive salience? Brain Res. Rev., 28, 309-369.

Berridge, K.C. \& Valenstein, E.S. (1991) What psychological process mediates feeding evoked by electrical stimulation of the lateral hypothalamus? Behav. Neurosci., 105, 3-14. 
Corbit, L.H. \& Balleine, B.W. (2003) Instrumental and Pavlovian incentive processes have dissociable effects on components of a heterogeneous instrumental chain. J. Exp. Psych.: Anim. Behav. Proc., 29, 99-106.

Critchfield, T.S. \& Kollins, S.H. (2001) Temporal discounting: basic research and the analysis of socially important behavior. J. Appl. Behav. Anal., 34, $101-122$.

Dayan, P. \& Balleine, B.W. (2002) Reward, motivation and, reinforcement learning. Neuron, 36, 285-298.

Di Chiara, G. (2002) Nucleus accumbens shell and core dopamine: differential role in behavior and addiction. Behav. Brain Res., 137, 75-114.

Dickinson, A. \& Balleine, B. (2002) The role of learning in the operation of motivational systems. In Gallistel, C.R. (Ed.), Stevens' Handbook of Experimental Psychology: Learning, Motivation, and Emotion. Wiley and Sons, New York, pp. 497-534.

Everitt, B.J. \& Wolf, M.E. (2002) Psychomotor stimulant addiction: a neural systems perspective. J. Neurosci., 22, 3312-3320.

Galaverna, O.G., Seeley, R.J., Berridge, K.C., Grill, H.J., Epstein, A.N. \& Schulkin, J. (1993) Lesions of the central nucleus of the amygdala. I: Effects on taste reactivity, taste aversion learning and sodium appetite. Behav. Brain Res., 59, 11-17.

Ghitza, U.E., Fabbricatore, A.T., Prokopenko, V., Pawlak, A.P. \& West, M.O. (2003) Persistent cue-evoked activity of accumbens neurons after prolonged abstinence from self-administered cocaine. J. Neurosci., 23, 7239-7245.

Giordano, L.A., Bickel, W.K., Loewenstein, G., Jacobs, E.A., Marsch, L. \& Badger, G.J. (2002) Mild opioid deprivation increases the degree that opioiddependent outpatients discount delayed heroin and money. Psychopharmacology, 163, 174-182.

Harmer, C.J. \& Phillips, G.D. (1998) Enhanced appetitive conditioning following repeated pretreatment with d-amphetamine. Behav. Pharmacol., 9, 299-308.

Hyman, S.E. \& Malenka, R.C. (2001) Addiction and the brain: the neurobiology of compulsion and its persistence. Nat. Rev. Neurosci., 2, 695-703.

Kalivas, P.W., Churchill, L. \& Romanides, A. (1999) Involvement of the pallidal-thalamocortical circuit in adaptive behavior. Ann. N.Y. Acad. Sci., 877, 64-70.

Kalivas, P.W. \& Nakamura, M. (1999) Neural systems for behavioral activation and reward. Curr. Opin. Neurobiol., 9, 223-227.

Kelley, A.E., Andrzejewski, M.E., Baldwin, A.E., Hernandez, P.J. \& Pratt, W.E. (2003) Glutamate-mediated plasticity in corticostriatal networks: role in adaptive motor learning. Ann. N.Y. Acad. Sci., 1003, 159168.

Klitenick, M.A., Deutch, A.Y., Churchill, L. \& Kalivas, P.W. (1992) Topography and functional role of dopaminergic projections from the ventral mesencephalic tegmentum to the ventral pallidum. Neuroscience, $\mathbf{5 0}$, 371-386.

Kuczenski, R., Melega, W.P., Cho, A.K. \& Segal, D.S. (1997) Extracellular dopamine and amphetamine after systemic amphetamine administration: comparison to the behavioral response. J. Pharmacol. Exp. Ther., 282, 591596.

MacLennan, A.J. \& Maier, S.F. (1983) Coping and the stress-induced potentiation of stimulant stereotypy in the rat. Science, 219, 1091-1093.

Marinelli, M., Cooper, D.C., Baker, L.K. \& White, F.J. (2003) Impulse activity of midbrain dopamine neurons modulates drug-seeking behavior. Psychopharmacology, 168, 84-98.

Matthews, T.J. \& Lerer, B.E. (1987) Behavior patterns in pigeons during autoshaping with an incremental conditioned-stimulus. Learn. Behav., 15, 69-75.

McClure, S.M., Daw, N.D. \& Montague, P.R. (2003) A computational substrate for incentive salience. Trends Neurosci., 26, 423-428.

Montague, P.R. \& Berns, G.S. (2002) Neural economics and the biological substrates of valuation. Neuron, 36, 265-284.

Nicola, S.M., Yun, I.A., Wakabayashi, K.T. \& Fields, H.L. (2004) Cueevoked firing of nucleus accumbens neurons encodes motivational significance during a discriminative stimulus task. J. Neurophysiol., 91, $1840-1865$.

O’Doherty, J.P., Dayan, P., Friston, K., Critchley, H. \& Dolan, R.J. (2003) Temporal difference models and reward-related learning in the human brain. Neuron, 38, 329-337.

O'Donnell, P. (2003) Dopamine gating of forebrain neural ensembles. Eur. J. Neurosci., 17, 429-435.

Olausson, P., Jentsch, J.D. \& Taylor, J.R. (2003) Repeated nicotine exposure enhances reward-related learning in the rat. Neuropsychopharmacology, 28, $1264-1271$.
Paulson, P.E., Camp, D.M. \& Robinson, T.E. (1991) Time course of transient behavioral depression and persistent behavioral sensitization in relation to regional brain monoamine concentrations during amphetamine withdrawal in rats. Psychopharmacologia, 103, 480-492.

Peoples, L.L., Uzwiak, A.J., Gee, F. \& West, M.O. (1999) Tonic firing of rat nucleus accumbens neurons: changes during the first 2 weeks of daily cocaine self-administration sessions. Brain Res., 822, 231-236.

Phillips, G.D., Harmer, C.J. \& Hitchcott, P.K. (2002) Blockade of sensitisationinduced facilitation of appetitive conditioning by post-session intraamygdala nafadotride. Behav. Brain Res., 134, 249-257.

Pierce, R.C. \& Kalivas, P.W. (1997) A circuitry model of the expression of behavioral sensitization to amphetamine-like psychostimulants. Brain Res. Brain Res. Rev., 25, 192-216.

Redish, A.D. (2004) Addiction as a computational process gone awry. Science, 306, 1944-1947.

Robinson, T.E. \& Berridge, K.C. (1993) The neural basis of drug craving an incentive-sensitization theory of addiction. Brain Res. Rev., 18, $247-$ 291.

Robinson, T.E. \& Berridge, K.C. (2003) Addiction. Annu. Rev. Psychol., 54, $25-53$.

Robinson, T.E. \& Kolb, B. (1999) Alterations in the morphology of dendrites and dendritic spines in the nucleus accumbens and prefrontal cortex following repeated treatment with amphetamine or cocaine. Eur. J. Neurosci., 11, 1598-1604.

Robinson, S., Sandstrom, S.M., Denenberg, V.H. \& Palmiter, R.D. (2005) Distinguishing whether dopamine regulates liking, wanting, and/or learning about rewards. Behav. Neurosci., 119, 5-15.

Roitman, M.F., Na, E., Anderson, G., Jones, T.A. \& Bernstein, I.L. (2002) Induction of a salt appetite alters dendritic morphology in nucleus accumbens and sensitizes rats to amphetamine. J. Neurosci., 22, RC225RC229.

Schultz, W. (2002) Getting formal with dopamine and reward. Neuron, 36, 241-263.

Schultz, W., Dayan, P. \& Montague, P.R. (1997) A neural substrate of prediction and reward. Science, 275, 1593-1599.

Smith, K.S. \& Berridge, K.C. (2005) The ventral pallidum and hedonic reward: neurochemical maps of sucrose 'liking' and eating. J. Neurosci., 25, 8637-8649.

Steiner, J.E., Glaser, D., Hawilo, M.E. \& Berridge, K.C. (2001) Comparative expression of hedonic impact: affective reactions to taste by human infants and other primates. Neurosci. Biobehav. Rev., 25, 53-74.

Tang, X.C., McFarland, K., Cagle, S. \& Kalivas, P.W. (2005) Cocaine-induced reinstatement requires endogenous stimulation of mu-opioid receptors in the ventral pallidum. $J$. Neurosci., 25, 4512-4520.

Taylor, J.R. \& Jentsch, J.D. (2001) Repeated intermittent administration of psychomotor stimulant drugs alters the acquisition of Pavlovian approach behavior in rats: differential effects of cocaine, d-amphetamine and 3,4-methylenedioxymethamphetamine ('Ecstasy'). Biol. Psychiat., 50, 137-143.

Timberlake, W., Wahl, G. \& King, D. (1982) Stimulus and response contingencies in the misbehavior of rats. J. Exp. Psych.: Anim. Behav. Proc., 8, 65-85.

Tindell, A.J., Berridge, K.C. \& Aldridge, J.W. (2004) Ventral pallidal representation of pavlovian cues and reward: population and rate codes. J. Neurosci., 24, 1058-1069.

Toates, F. (1986) Motivational Systems. Cambridge University Press, Cambridge.

Turner, M.S., Lavin, A., Grace, A.A. \& Napier, T.C. (2001) Regulation of limbic information outflow by the subthalamic nucleus: excitatory amino acid projections to the ventral pallidum. J. Neurosci., 21, 2820-2832.

Turner, M.S., Mignon, L. \& Napier, T.C. (2002) Alterations in responses of ventral pallidal neurons to excitatory amino acids after long-term dopamine depletion. J. Pharmacol. Exp. Ther, 301, 371-381.

Usuda, I., Tanaka, K. \& Chiba, T. (1998) Efferent projections of the nucleus accumbens in the rat with special reference to subdivision of the nucleus: biotinylated dextran amine study. Brain Res., 797, 73-93.

Vuchinich, R.E. \& Simpson, C.A. (1998) Hyperbolic temporal discounting in social drinkers and problem drinkers. Exp. Clin. Psychopharmacol., 6, 292305.

Wyvell, C.L. \& Berridge, K.C. (2001) Incentive sensitization by previous amphetamine exposure: increased cue-triggered 'wanting' for sucrose reward. J. Neurosci., 21, 7831-7840.

Zhang, J., Riehle, A., Requin, J. \& Kornblum, S. (1997) Dynamics of single neuron activity in monkey primary motor cortex related to sensorimotor transformation. J. Neurosci., 17, 2227-2246. 